\title{
THE
}

\section{Towards Fog-driven loT eHealth: Promises and Challenges of IoT in Medicine and Healthcare}

\author{
Bahar Farahani \\ Farshad Firouzi \\ Victor Chang \\ Mustafa Badaroglu \\ Nicholas Constant \\ University of Rhode Island \\ Follow this and additional works at: https://digitalcommons.uri.edu/ele_facpubs \\ The University of Rhode Island Faculty have made this article openly available. \\ Please let us know how Open Access to this research benefits you.
}

This is a pre-publication author manuscript of the final, published article.

Terms of Use

This article is made available under the terms and conditions applicable towards Open Access

Policy Articles, as set forth in our Terms of Use.

\section{Citation/Publisher Attribution}

Farahani, B., Firouzi, F., Chang, V., Badaroglu, M., Constant, N., \& Mankodiya, K. (2018). Towards Fog-driven IoT eHealth: Promises and Challenges of loT in Medicine and Healthcare. Future Generation Computer Systems, 78(2), 659-676. doi: 10.1016/j.future.2017.04.036

Available at: https://doi.org/10.1016/j.future.2017.04.036

This Article is brought to you for free and open access by the Department of Electrical, Computer, and Biomedical Engineering at DigitalCommons@URI. It has been accepted for inclusion in Department of Electrical, Computer, and Biomedical Engineering Faculty Publications by an authorized administrator of DigitalCommons@URI. For more information, please contact digitalcommons-group@uri.edu. 


\section{Authors}

Bahar Farahani, Farshad Firouzi, Victor Chang, Mustafa Badaroglu, Nicholas Constant, and Kunal Mankodiya

This article is available at DigitalCommons@URI: https://digitalcommons.uri.edu/ele_facpubs/79 


\title{
Towards Fog-driven IoT eHealth: Promises and Challenges of IoT in Medicine and Healthcare
}

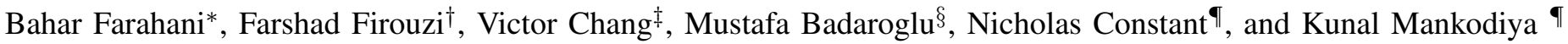 \\ ${ }^{*}$ School of Electrical and Computer Engineering, University of Tehran \\ ${ }^{\dagger}$ Department of Electrical, and Computer Engineering, KU Leuven \\ ${ }_{\ddagger}^{\ddagger}$ International Business School Suzhou, Xi’an Jiaotong Liverpool University, Suzhou, China \\ $\S$ Qualcomm Technologies \\ I Department of Electrical, Computer, and Biomedical Engineering, University of Rhode Island
}

\begin{abstract}
Internet of Things (IoT) offers a seamless platform to connect people and objects to one another for enriching and making our lives easier. This vision carries us from compute-based centralized schemes to a more distributed environment offering a vast amount of applications such as smart wearables, smart home, smart mobility, and smart cities. In this paper we discuss applicability of IoT in healthcare and medicine by presenting a holistic architecture of IoT eHealth ecosystem. Healthcare is becoming increasingly difficult to manage due to insufficient and less effective healthcare services to meet the increasing demands of rising aging population with chronic diseases. We propose that this requires a transition from the clinic-centric treatment to patient-centric healthcare where each agent such as hospital, patient, and services are seamlessly connected to each other. This patient-centric IoT eHealth ecosystem needs a multi-layer architecture: 1) device, 2) fog computing and 3) cloud to empower handling of complex data in terms of its variety, speed, and latency. This fog-driven IoT architecture is followed by various case examples of services and applications that are implemented on those layers. Those examples range from mobile health, assisted living, e-medicine, implants, early warning systems, to population monitoring in smart cities. We then finally address the challenges of IoT eHealth such as data management, scalability, regulations, interoperability, device-network-human interfaces, security, and privacy.
\end{abstract}

\section{INTRODUCTION}

Internet of Things (IoT) is an ever-growing ecosystem that integrates hardware, computing devices, physical objects, softwares, and animals or people over a network enabling them to interact, communicate, collect and exchange data. There is an increased number of users, services and applications associated with IoT across different disciplines [1], [2]. IoT has been evolved from Radio Frequency Identification (RFID) [3] and Wireless Sensor Network (WSN) technologies [4], [5], [6], [7], [8], [9] to more advanced integration with cloud computing, Internet services, cyber-physical systems and interconnections between hardware and software devices [3]. A typical IoT system consists of sensors, communication interfaces, advanced algorithms, and cloud interface. Sensors are used to collect data from different devices. RFID technology and WSN technologies provide the means of communications and network infrastructure. Advanced algorithms are used to process data and analyze anything meaningful through Application Program Interfaces (APIs) or apps. Thousands of client-server requests can be exchanged between mobile devices and services in the cloud and Internet, thus allowing users to get access to different types of services at the same time [1], [2], [10], [11].

There are different major types of IoT services as follows [1], [10], [12], [13], [14], [15]. First, smart wearable devices can be used for patients who need to collect data about their health status such as heartbeat, blood pressure and glucose level through sensors on the wearable technologies, which are sent to smartphones. The health status of patients can be monitored at the same time. Second, smart homes can be enhanced by IoT. While sensors can detect the changes in temperature, air-conditioning systems can be monitored. Home security cameras can capture any intruders and send the warnings to the home owners by mobile apps. Third, traffic and transport systems can be monitored by IoT to achieve smart cities. Data can be collected and analyzed to understand the updates in traffic networks and transportation systems. Forth, supply chain system can work with IoT that all the deliveries can be recorded and tracked in real-time. When goods have been shipped to the destination, the delivery records can be kept up-to-date. Fifth, the Internet banking can work with trustworthy third-party payment systems on the smartphones. Examples include Apple Pay, PayPal, Taobao and Alibaba that customers can purchase and pay within seconds. Customers can have more varieties of options for their purchase and have a more convenient way to complete their transactions more easily without a need to go the vendor directly. These use cases of IoT can be mapped to a generic model where this generic model is to provide an easier integration with different services seamlessly, ranging from sensors to network infrastructure, and from APIs to big data processing, and finally from analytics to predictive modeling [1], [16], [17]. This approach ensures that users can have easier access to services, can monitor the progress of the service delivery, and can compare prices and ratings offered by service providers. Moreover, service providers can be more exposed to more customers, check their business performance and track the market trend more easily [10], [16], [17]. 
In general, IoT has a multi-layer architecture that can be divided into four distinct levels (See Fig. 1) [10]. From the bottomup view, the first layer is sensing layer, which integrates with all different types of hardware connect to the physical world and collect data. The second layer is networking layer, which offers networking support and data transfers in the wired and wireless networks. The third layer is the service layer that creates and manages all types of services aiming to satisfy user requirements. The top layer is the interface layer, which offers interaction methods to users and other applications, so that all the data can be analyzed and all the outputs can be presented and delivered smoothly.

The interaction between technology and healthcare has a long history. However, the rapid growth of IoT as well as the public embracement of miniature wearable bio-sensors have generated new opportunities for personalized eHealth and services. In this context, the above-mentioned four-layered concept is applicable to healthcare technologies and services, particularly the example of smart healthcare services and smart wearable devices as follows. Smart healthcare services can collect different types of data in the sensing layer through sensors on cyber-physical or wearable systems. All the data can be transported to the cloud or servers that collect all the data through wireless networks in the networking layer. The service layer provides the actual healthcare service, such as processing the patients data about their health status involved with heartbeat, blood pressure and glucose level. The interface layer is to provide an easier interface that allows users to retrieve the outputs and to understand meanings of the outputs can be received on both patients and doctors, so that doctors can diagnose when next appointment can be made. Patients can understand quickly about their health status without spending more time and money for medical checkups.

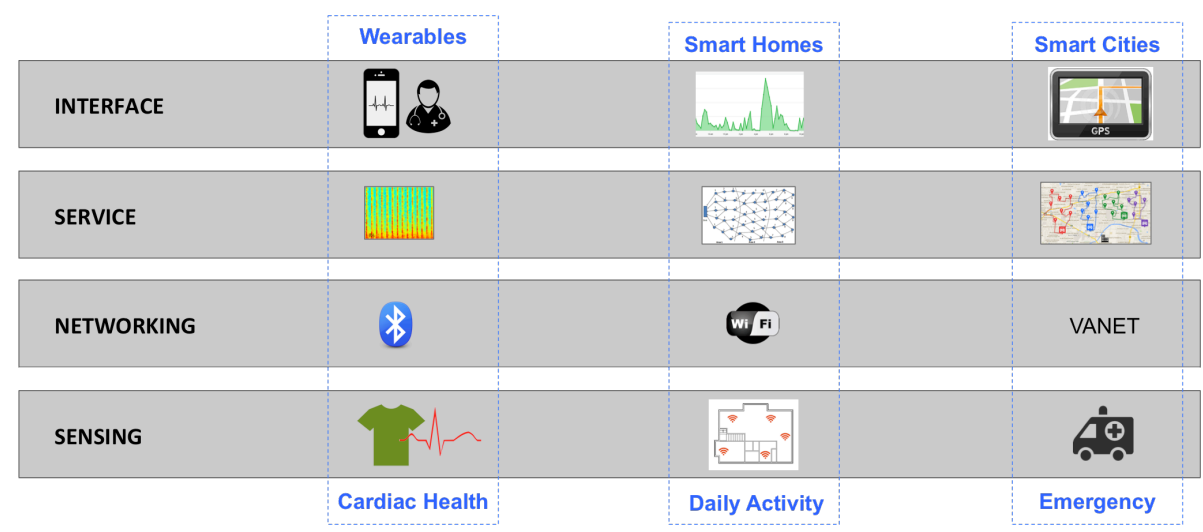

Fig. 1: IoT layers.

Additionally, IoT eHealth can work seamlessly with security since it is a challenge to retain a high level of security and privacy for healthcare sector as follows [12], [13], [14], [15]. First, RFID and WSN technologies can be used to provide identification and tracking technologies, so that each device for each user can be identified and tracked by barcode and intelligent sensors. Second, network authentication and network firewall can be provided to ensure a strong network security. Third, IoT eHealth services can offer authentication, authorization, confidentiality and integrity for all data and IoT services. All the passwords will be authenticated and double checked with the identity on the barcode on the hardware and personal keys on the software to maximize the level of protection. On the top layer, each user identity and location privacy can be kept anonymous to minimize the possibility of leakage of personal information on IoT. This concept is similar to the multi-layered security model demonstrated by [18], whereby different types of security services have been integrated based on results of three years of implementations and testing.

With examples described above, benefits of adopting IoT eHealth can be summed up as follows [1], [2], [19], [10], [16], [17], [20], [12], [14], [13]:

- All-encompassing: whether people use IoT eHealth for health, exercise, safety or beauty reasons, it has a holistic solution for everyone needs.

- Seamless fusion and integration with different technologies: IoT eHealth enables different technologies to work together seamlessly without concerning the complexity of technology integration.

- Big Data processing and analytics: IoT eHealth can effectively process, analyze, and manipulate the tsunami of multi-scale, multi-modal, distributed and heterogeneous data sets produced by connected sensors in a fair amount of time. This allows to extract useful actionable information from health data.

- Ability to personalize and tailor content/service: IoT and big data analytics can vastly expand the possibilities to fulfill the necessity of personalized healthcare and treatments. Therefore, it plays a notable role in the well-being. For example, machine learning and big data analytics can be used to forecast health conditions (e.g., cancers, infections, and heart 
attacks) before they occur. As a result, patients can take quick actions.

- Lifetime monitoring: patients can receive comprehensive additive data on their past, present, and future health.

- Easy to use: IoT eHealth can be easily adopted by users since they only require clicks on wearable devices and/or some simple operations on smartphone apps.

- Cost reduction: there are two types of cost reduction involved. First, the cost reduction is achieved due to the technological fusion and there is no need to pay separate payment for the use of particular technology. Second, patients can monitor their health status which makes them consult doctors when health status is only below the recommendation.

- Doctors can be more involved: since doctors can receive data about patients health status in real-time, it saves them effort to examine patients. Doctors can also monitor a higher number of patients by relying on the healthcare IT systems. However, the organizational structures hosting the clinicians may be required to evolve in order to best take advantage of the real-time data available with telemedicine.

- Availability and accessibility: patients and those engaged in the patients care can have access to eHealth data/services anytime, anywhere without any geographical barriers.

- Online assistance: IoT eHealth enables real-time, 24/7 online access to health specialists such as doctors, dermatologists and many other professionals.

- Better efficiencies for healthcare resource management: IoT ensures patients to easily know their health status and doctors to easily monitor patients health. IoT also results in better efficiencies to manage healthcare resources.

- International collaboration: health professionals around the world are connected via IoT eHealth ecosystem. This enables patients to have more access to international facilities at their fingertips (anywhere and anytime).

There is an increasing number of publications for IoT and IoT for healthcare as follows. While extracting the number of publications from Google Scholar, Fig. 2 shows the number of publications for the terms IoT and IoT for healthcare between Year 2011 and Year 2015. The number of publications for the term IoT has decreased annually from 134,000 in Year 2011 to 75,500 in Year 2014, and it has surged again to 97,300 IoT publications in Year 2015. On the other hand, for the category of IoT for Healthcare, the extent of publications increase has been gradual, from 23,200 in Year 2011 to 30,900 in Year 2015. Our publication extraction shows that despite of a large number of IoT publications, there is still a demand to increase outputs from IoT for healthcare. While acknowledging the benefits of IoT for healthcare, more research outputs will be making contributions to the development of healthcare sector.

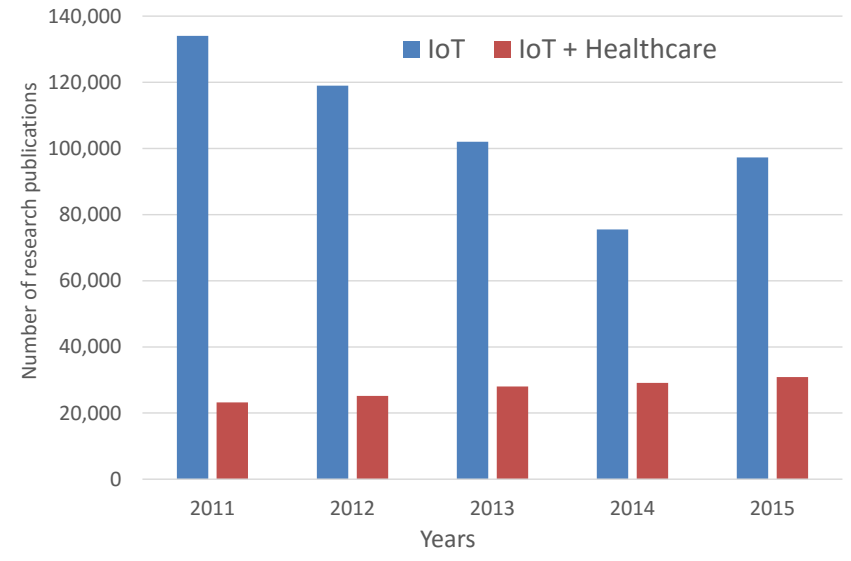

Fig. 2: IoT popularity index.

In the subsequent sections, we will deeply discuss how IoT could benefit the healthcare systems across the globe and what challenges and barriers IoT has to overcome to grow further. The paper ends with the discussions of some of our ongoing activities that demonstrate the capabilities of IoT in medical domain.

\section{PRomises AND Visions of IOT in HEALTHCARE}

\section{A. Challenges of Today's Healthcare}

Most of the worlds regions are facing a formidable challenge to manage the rapidly increasing aging population, individuals with chronic conditions, child mortality, frequent outbursts of disease epidemics, and harsh living environments with poor sanity, lack of drinking water resources and rising pollution. Although, the world's demand for medical services has risen in recent years, we still live in the traditional model of hospital-centric care, in which citizens visit doctors when they fall sick. To manage their chronic disease, patients make frequent visits to hospitals or clinics for the doctors to make observations that include monitoring disease progression and also to make clinical decisions that lead to treatment adjustments. In general, 
hospitals use a disease- and physician-centered model that is more reactive and do not involve patients as an active part of the medical process. We briefly enumerate some of the challenges and barriers faced by hospital-centric medical practices:

- Limited time and prospective: increasing population with illnesses and disabilities limits the physicians to check each patient with a quality time. Due to the short screening time, physicians lack the prospective of patient's daily routine such as physical activity, diet, sleep, and social life, all of these attributes are equally important in the process of diagnosis and treatment.

- Adherence monitoring: physicians are not better equipped to check if their patients are adhering to prescribed treatment that may include medications, rehabilitation exercises, and some preventive activities such as avoiding some diet. It is quite common that the lack of adherence raises the risk of hospitalization [21] and hence, increases the economical burden to the patients and their families.

- Aging population: globally, the number of old adults (over 60 years of age) is expected to grow more than double, from 841 million individuals in 2013 to more than 2 billion in 2050 [22]. Thus, elderly population will seek more facilities and resources for medical care.

- Urbanization: WHO predicts that by $2015,70 \%$ of world's population will live in urban environments [23]. This implies that big cities will demand more healthcare infrastructure to serve rising population. Moreover, big cities will become a center of epidemic outbursts of contagious diseases that will spread easily across dense populations.

- Shortage of healthcare workforce: as the demand for healthcare services increases, it equally increases the demand for healthcare workers including physicians, surgeons, dentists, nursing staff, nurse assistants, professional caretakers, and laboratory staff who could enhance the healthcare ecosystem in urban and rural areas. One of the alternative to this challenge is to increase the infrastructure of telemedicine (described in the subsequent sections).

- Rising medical cost: one of the biggest factors in the healthcare today is the increasing cost for the healthcare. For example, the spending for diabetes care in USA is skyrocketing that is estimated to be around $\$ 245$ billion in 2016, up by $21 \%$ from 2007 [24].

\section{B. IoT-driven Healthcare: Transitioning from Hospital-Centric to Patient-Centric}

One of the emerging models for healthcare today is Patient-Centered Care (PCC) that was coined by Picker/Commonwealth Program developed by the Picker Institute in 1988. This model focuses on the patients and their individual healthcare needs. The 2001 landmark report [25] from Institute of Medicine further defined the PCC as following:

"Healthcare that establishes a partnership among practitioners, patients, and their families (when appropriate) to ensure that decisions respect patients wants, needs, and preferences and that patients have the education and support they need to make decisions and participate in their own care."

PCC asks patients to be partners in their own care. Despite many initiatives that provide supporting evidence of PCC and its success in smaller-to-medium scales, we are not even close to realizing PCC in a real-sense because hospital-centered model co-exists and conflicts with PCC. However, the good news is that PCC is not designed to eliminate hospitals and clinics. Instead PCC leverages them in the shared model for patient care. In order to truly integrate hospitals or clinics with patients in PCC, there is a need to utilize the powerful ecosystem of IoT. As we described earlier, IoT is a convergence of sensors, actuators, telecommunication, cloud computing and big data, interconnecting them through the Internet to provide goal-oriented services. Although IoT is a paradigm shift framework, fundamentally it is defined by an application. Hence, IoT could be tailored to address the challenges of healthcare.

Fig. 3 demonstrates the classification of the healthcare domain, dividing into three areas: i) large healthcare organizations such as hospitals, ii) small clinics and dispensaries, and iii) non-clinical environments such patients' homes, communities, nursing homes, and rural areas without any medical support. To envision IoT's role in the healthcare industry, it is important to identify the operations of each of these areas. When IoT could make a specific operation cost-efficient, reliable and timely, it would eventually evolve to impact other operations, designing a self-sustainable ecosystem for today's struggling medical care. In the following, we will take a tour of potentials areas where IoT technologies could play an instrumental role:

- Hospitals: hospitals are becoming bigger with resources to admit, treat, operate or monitor increasing number of patients. In order to run their operations smoothly, they constantly rely on advanced technologies. For example, smart ambulances could perform on-fleet diagnosis such that medical staff can make possible arrangements before the arrival of the patient. Such scenario can be conceived with the integration of IoT. Smart ambulances will need a set of reliable medical sensors for diagnosis, a secure communication link with hospital, and intelligent workflow management tools for hospital preparations that may include an operating room, where a surgeon knows about the condition of the patient well in advance. Surgeries could become more contextual and efficient with IoT when the information exchange among doctors, medical staff, and medical devices in operating room orchestrate with one another smoothly. Similarly, one can envision the role of IoT in other divisions of the hospital such as intensive care unit (ICU), primary care unit, and specialized units.

- Clinics: for the primary care, patients go to their family physicians who now could take advantage of IoT in many different ways. For example, the doctors could virtually access the lab reports of their patients before they visit. Clinic receptions 


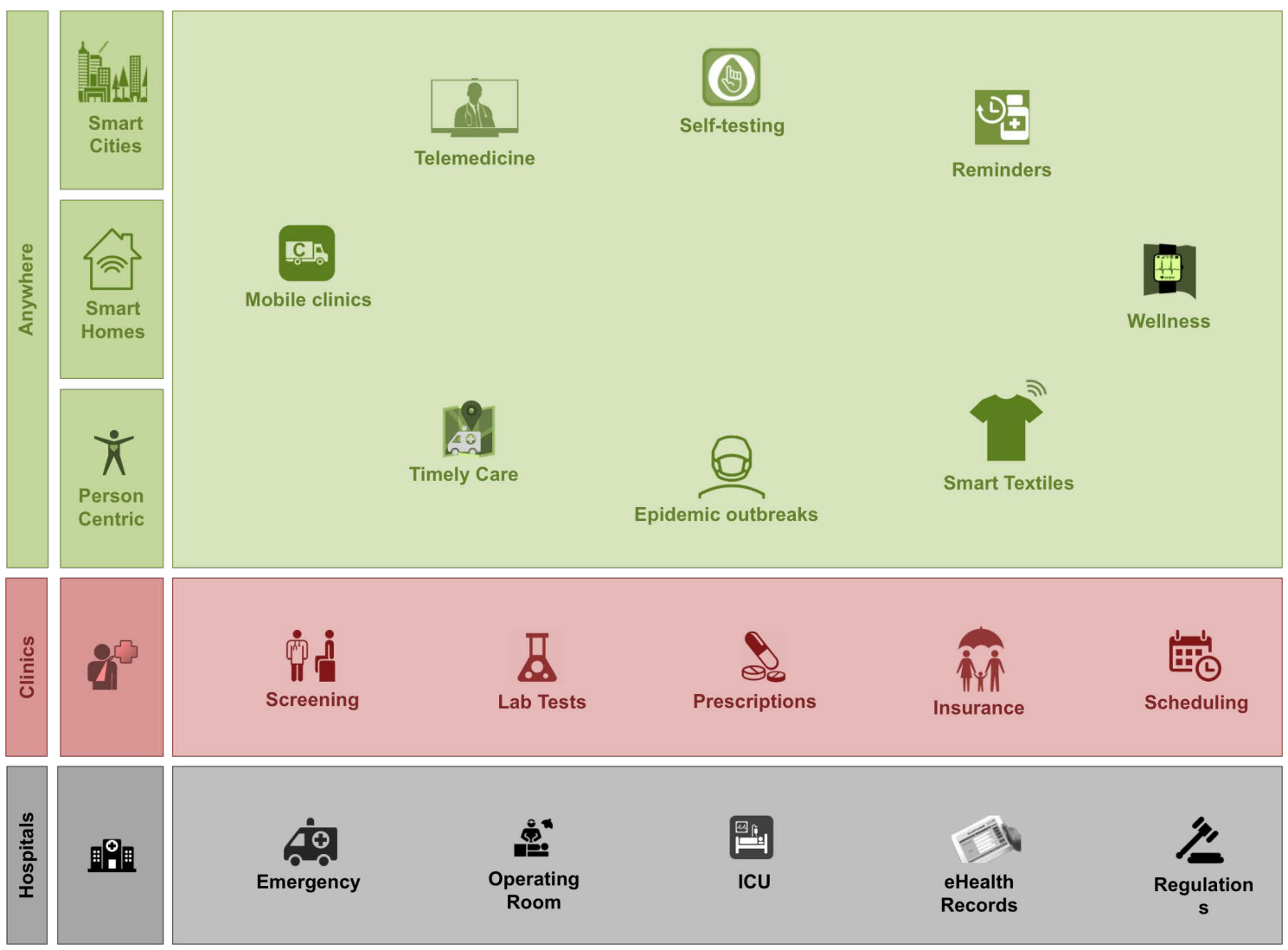

Fig. 3: Classifications of the medical and healthcare industry in the context of functions, operations, and applications. In each of the visualized domains and subdomains, IoT could play an important role to make the medical processes and procedures more cost efficient, timely, reliable, and easy.

could check the insurance coverage in real-time. IoT could provide a secure bi-directional communication between patients and medical receptions to set appointments without making long phone calls. IoT could provide a low-cost solution for clinics to have a connected screening for their patients so the physician could spend more quality time with patients. Physicians could prescribe inexpensive IoT devices for patients to take home for self-testing that could lead the way towards PCC.

- Anywhere (Non-clinical Settings): lots of excitement today is about the role of IoT in remote or telehealth care, in which patients are expected to gain tremendous benefits:

- Mobile clinics: there is an increasing interest in mobile health clinics across the globe. For example, in African countries, mobile clinics are proved to offer a low-cost, high quality care for vulnerable populations in remote areas where citizens have no access to basic medical facilities [26]. However, mobile clinics are vehicles with limited medical facilities. IoT could make a big difference in enhancing the infrastructure of the mobile clinics that could partner with large hospitals to remotely communicate for diagnosis and decision support. In this way, mobile clinics could scale up their capabilities for care.

- Telemedicine: without a doubt, the telemedicine is one of the first and foremost stakeholders of IoT today. According to a recent report [27], the global market for telemedicine is estimated to be worth around $\$ 23,224$ million and is expected to go up around $\$ 66,606$ million by 2021 with an estimated growth of $18.8 \%$. IoT has a lot to offer in this field because a large portion of world's population owns cellphones and smartphones, which are not mere communication devices, but also used for connecting with other sensors. In the case of medical care, smartphones are playing a vital role by enabling patients to self-test their own health. The self-test data become important predictors of health and diseases. Moreover, physicians and medical staff have ways to see their patients virtually via teleconferencing. IoT could further augment telemedicine by networking sensors to facilitate tele-screening. In this way, the hospitals still could survive with less number of healthcare workers by increasing the virtual care services.

- Wellness: the old proverb "an ounce of prevention is worth a pound of cure" is so true and fits very appropriately with the current state of healthcare. World's population is becoming more aware that it is better to take preventive 
actions and be more proactive about one's health than living with medicines, pain, and suffering later in the life. Global citizens are turning towards advanced technologies such as wearable trackers, sports tech, and self-testing medical devices which are also categorized as IoT devices because they provide connected, continuous monitoring of one's health and wellness. IoT could help build an economical scheme for insurance coverage to curb the healthcare cost based on wellness rewards, generated from the health tracking. This could be one of many ways to promote global populations to be partners of healthcare system, establishing a more efficient patient-centered care.

- Smart homes: while the telemedicine services can reduce the number of visits to hospitals and clinics, especially for patients who are elderly with chronic conditions or disabilities, it becomes vital to establish a technical infrastructure in their homes. Hence, there needs to be smart homes and environments, providing unobtrusive support systems to enable citizens to experience independence, activity, quality of life or well-being. In Europe, Ambient Assisted Living, a successful smart homes framework, is conceptualized as ambient intelligence aimed at improving peoples capabilities using sensitive, adaptive, and responsive digital environments [28], [29], [30]. In general, IoT has penetrated the market of home automation to provide interactive capabilities for residents to control the appliances using smartphones and remote controls [31]. Furthermore, IoT could be customized to fulfill specific healthcare needs of elderly individuals. Smart toilets could execute urine testing on regular basis for preventive and primary care. Smart homes could adjust lights and sounds to avoid sensory overloads for individuals with autism who then could live better quality life.

- Smart cities and connected communities: the rapid migration of massive population to urban regions has raised the demands and challenges in the world's largest cities [23]. Hence, emerging efforts are made across the globe to build smart cities [32], [33] and connected communities [34] by integrating and connecting various infrastructures to improve livability, sustainability, affordability, and efficiency. IoT architectures are leveraged and customized for smart cities to provide the right information at the right place and at the right time to make a right decision to aid citizens more quickly [35], [36]. As the healthcare system is an integral part of cities and urban environments, it has been woven automatically into the fabrics of smart cities and communities in multiple ways. For example, cities have environment sensors distributed in their infrastructure, streaming the sensor data to a centralized location such the cloud servers that could now be accessed by the healthcare system. The access to the environment sensors could make citizens aware of the conditions such as pollutants, pollen, humidity, temperature, and water contamination. IoT could deliver this information in real-time to avoid and prevent health issues in advance. For instance, asthma patients could be alerted to avoid being in the regions with high particles (pollen or dust) [37]. With the same approach, it is possible to reduce the intensity of lung cancer [38]. Another example of how IoT-driven smart cities can make the healthcare more efficient is the convergence of smart traffic lights and ambulance routing to save lives with the cooperation of transportation infrastructure [39].

As we can see that IoT's future is promising in the areas of medicine, healthcare, and wellness. However, it demands an intelligent ecosystem that would allow interconnection among all stakeholders including patients, physicians (in clinics), large hospitals, cities and communities. In next section, we will describe how eHealth IoT ecosystem could be built by leveraging the new paradigm of Fog Computing.

\section{IOT EHEALTh ECOSYSTEM}

In this section, we explain a holistic multi-layer IoT eHealth ecosystem/architecture that can fundamentally change the way organizations and caregivers deliver wellness and health. As shown Fig. 4, this system consists of three main layers: IoT eHealth Device Layer, IoT eHealth Fog Layer and IoT eHealth Cloud Layer. Note that IoT architecture for eHealth applications is already discussed in [40], [41], [42], [43], [44], [45], [46], [47], [48]. However, the following provides a complete solution from data acquisition and processing, to cloud platform and big data analytics.

\section{A. IoT eHealth Device Layer}

This rich set of smart IoT medical devices enables individuals to monitor their health data any time, from any computer or mobile device (all in real-time) and sync their data securely with the cloud eHealth platform [19]. All they need to perform is to provide a connection using a suitable communication protocol to a gateway or a fog node. In this context, there is a vast variety of Personal Area Networks (PAN) and WSN protocols. Fig. 5 shows the IoT eHealth protocol stack. Note that selecting the best connectivity and the communication protocol highly depend on the application and the specific use-case. For example, to transfer a large amount of documents wirelessly, Wi-Fi is ideal. On the other hand, BLE best fits for short-range low power communications. The state-of-the-art IoT eHealth devices can be classified into two main groups:

- Physical sensor: generally any medical device with a wired/wireless interface can be used in eHealth ecosystem to track patients' physical wellness, and digitally monitor their health [49]. This includes ECG/EKG monitor [50], [51], [52], [53], [54], [55], [56], [57], [58], [59], [60], [61], heart rate monitor [62], [63], glucose monitor [64], [65], [66], blood pressure monitor [67], [68], [69], body temperature monitor [70], pulse oximeter [71], hemoglobin monitor [72], activity monitor [73], smart shoes [74], smart garments or e-textiles [75], [76], [77], sleep monitor [78], knee sensor [79], skin 


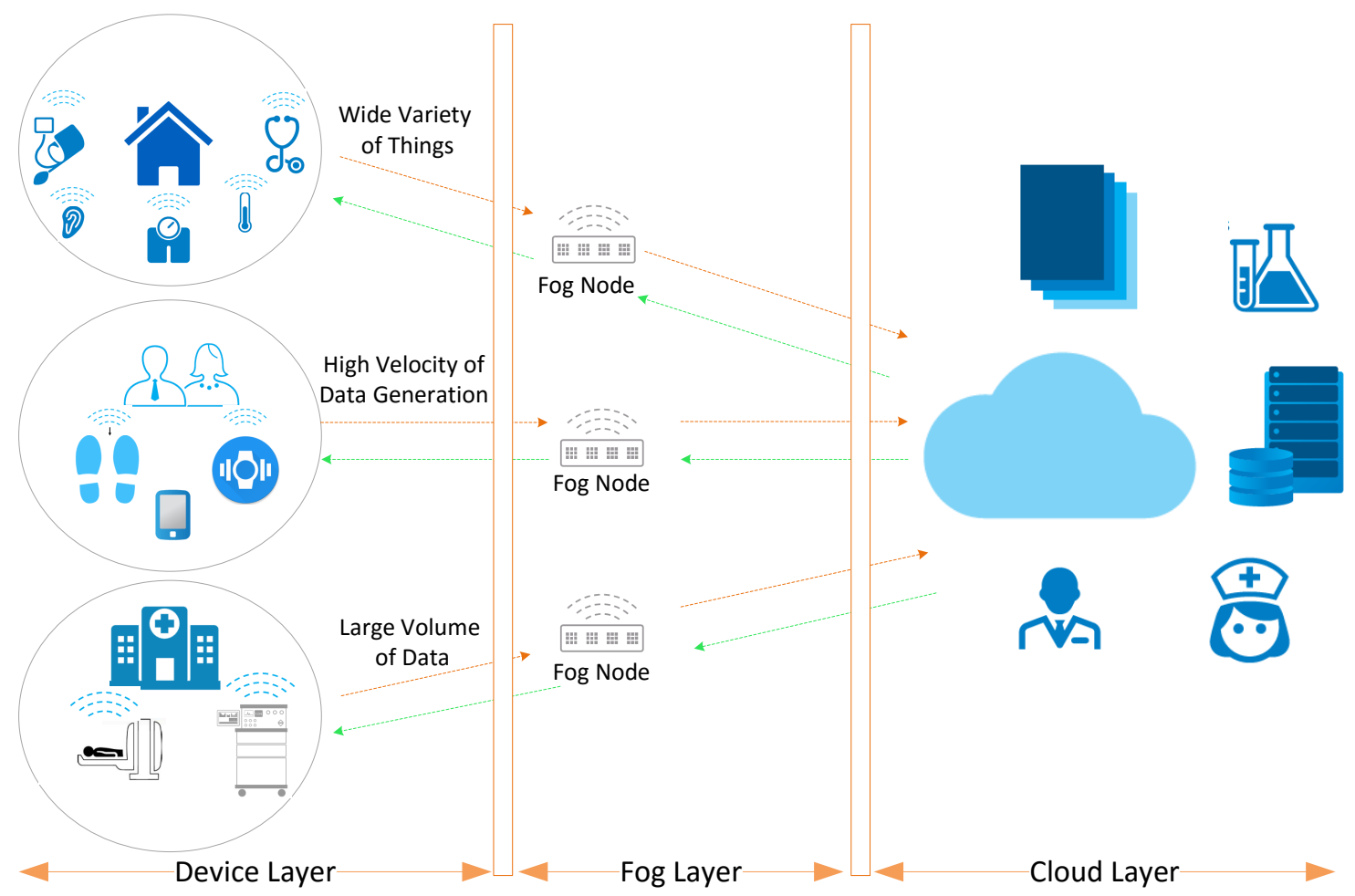

Fig. 4: IoT eHealth ecosystem / architecture.

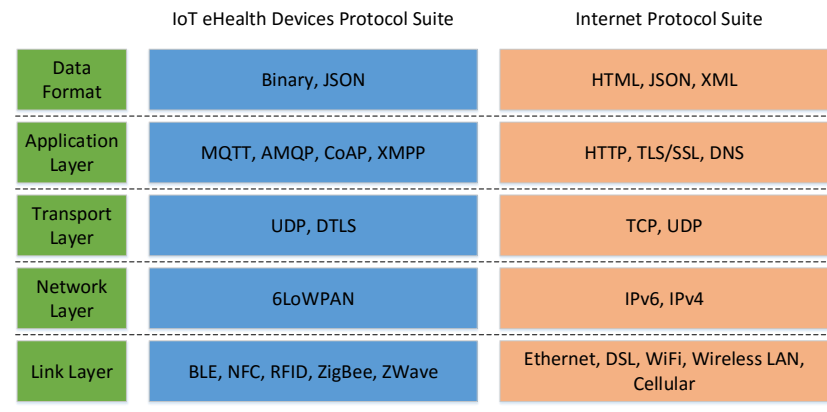

(a)

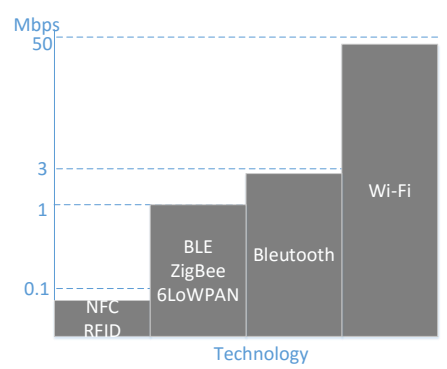

(b)

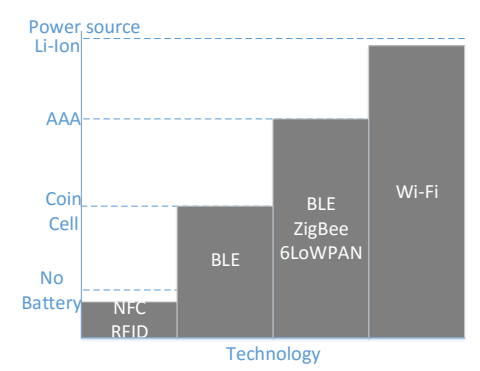

Fig. 5: (a) IoT eHealth protocol stack, (b) Maximum throughput and power source for various PAN and WSN [96].

conductance sensor [80], PPG [81], elderly monitor [82], [83], [84], [85], [86], medication management [87], [88], food contamination detection device [89], early warning system [90], [91].

- Virtual sensor: using software and mobile applications as well as eHealth services, virtual sensors capture patient's health data and contextual data from the environment [92], [93], [94], [95]. Virtual sensor includes many categories such as remote monitoring, remote consultation, diagnostic, patient health record, nutrition, and medical reference applications.

\section{B. IoT eHealth Fog Layer}

eHealth is among those critical IoT applications which cannot afford latency. Indeed, it is a necessity to be able to analyze and act on the time-sensitive data and circumstances such as Myocardial Infarction (MI) in seconds. Therefore, it is not practical to rely on the traditional cloud model and architecture to collect and analyze the patients' sensitive medical data, vital signs, and bio-signals across a wide geographical area in the presence of various environmental conditions. The most efficient approach to tackle this issue is using fog or edge computing to take the cloud computing and services to the next level. A fog node is defined as a device with computing, storage, and network connectivity. In the proposed eHealth platform, we analyze the time-sensitive data, and make the extremely time-sensitive decision on the fog nodes. These nodes are placed closest to the medical devices that produce the data. On the other hand, we send the rest of the data to the cloud as the main storage 
and computing resource. It should be noted that another main issue with IoT eHealth is to conserve the network bandwidth. For example, an ECG device can generate several GBs of raw time-series data within a day. However, it is not feasible and not even necessary to send these huge amounts of data from thousands of patients to the cloud. As a result, fog nodes are reasonable to process, filter, and compress the data traveling between the medical devices and the cloud. As shown in Fig. 6, the main features of the fog node are as follow:

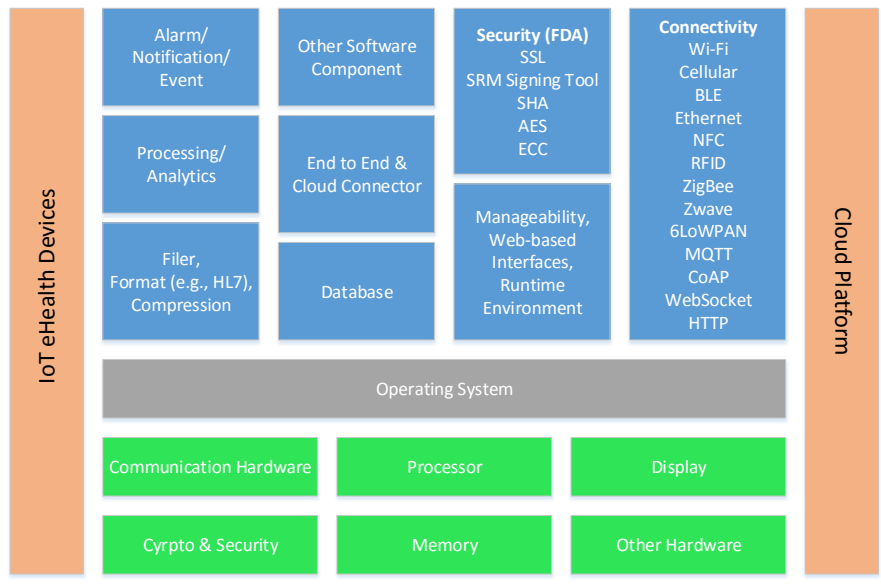

Fig. 6: Architecture of a fog node.

- Connectivity: fog nodes support wide-range of hardware interfaces and protocols. They can practically establish a communication with any eHealth devices as well as the cloud platform. The fog nodes are capable of delivering reliable and secure connectivity between devices, across networks and between networks. This includes protocol translation, security, switching, routing, and networking analytics.

- Two-way data exchange: fog nodes involve a bi-directional data flowing. The node periodically sends the aggregated medical data to the cloud for a long-term storage and big data analytics. It also receives the commands as well as the configuration data from the cloud. Since fog nodes might not have a valid public IP, it is important to ensure the reachability of the fog nodes from the cloud. To achieve this goal different mechanisms can be used such as WebSocket, MQTT (Message Queue Telemetry Transport), and IP tunneling.

- Flexible integration: fog nodes receive, collect and capture patients data from sensors and medical devices in real-time. However, a vast variety of medical devices, and instruments from different device vendors and OEMs are available. To be interoperable with this wide-range of medical devices and wide-range of interfaces, fog nodes are equipped with a multi-standard interfaces. These interfaces should be compatible with various PAN and WSN protocols (such as RFID, BLE, Zigbee, Wi-Fi, 3G/4G, and Ethernet), wired protocols (such as Ethernet) as well as different serial protocols (such as UART, SPI and USB).

- Protocol translation: similar to devices, there is a wide variety of protocols. Therefore, to be interoperable, the fog nodes perform the necessary protocol translation at different network layers. Note that IoT eHealth interoperability crisis can occur at different abstraction layers such as:

- Interoperability at network layer: IoT eHealth network is scattered among various low power networking protocols (such as BLE, ZigBee), and conventional networking protocols (such as Wi-Fi). One of the key tasks of fog nodes is to bridge between different networks by converting the incoming data to the correct format and sending out on the other network.

- Interoperability at messaging layer: there is a vast variety of application level protocols such as MQTT, CoAP (Constrained Application Protocol), and XMPP (Extensible Messaging and Presence Protocol). Each of them has a unique protocol for processing the messages. Therefore, it is very important that the fog nodes to be independent of the underlying message protocol standards and to be able to easily transfer messages between different protocols.

- Interoperability at data annotation layer: various organizations developed different standards for integration, exchange, and retrieval of Electronic Health Record (EHR) such as HL7 [97]. The fog nodes can process and re-format the health data, whenever it is necessary.

- Data aggregation, filtering, formatting, and encoding/decoding: the incoming data are from various sources such as ECG, weighing scales, blood pressure, glucose, pulse-oximeters, etc. Fog nodes perform filtering, aggregating, compressing, and formatting of the collected raw medical data to be interoperable and suitable for the other resources.

- Short-time database: the node typically can provide short time storage for the incoming data. 
- Security and data protection: to protect the patients data, the fog node offers multi-layer security measures for authentication, encryption and access control to fully meet the requirements of the FDA standards.

- Assessment and notification: fog nodes can evaluate and process the incoming raw data to determine if it requires to tagger an alert or notification. This can involve event generation and transforming the data/events to additional destinations.

- Local processing: depending on the requirements of the health applications and the specifications of the fog nodes, a rich set of applications can be executed. This includes data mining and pattern recognition in time-series data, feature extraction, machine learning, light-weight signal processing, event processing, automated reasoning, embedded web server, embedded WebSocket server, etc.

\section{IoT eHealth Cloud Layer}

The proposed cloud benefits of a multi-layer architecture and it consists of the following layers (See Fig. 7) [19]:

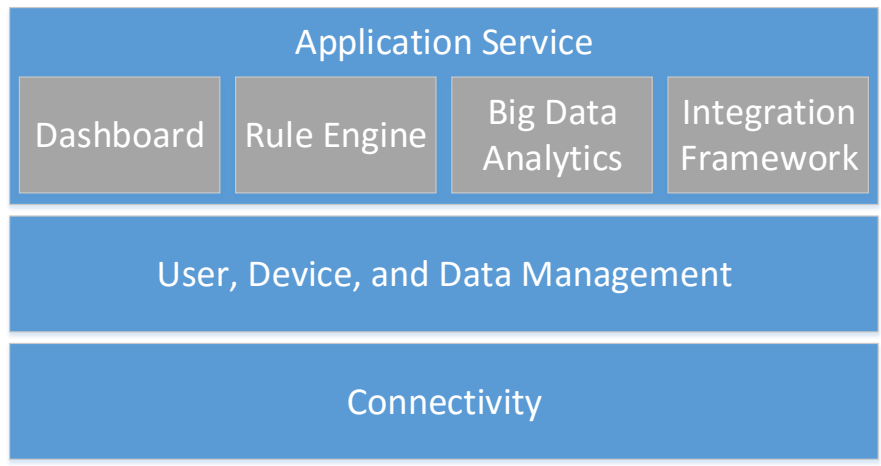

Fig. 7: Multi-layer architecture of the eHealth cloud.

- Connectivity: the connectivity module is equipped with a vast variety of built-in capabilities to establish connectivity between eHealth devices, fog nodes and the cloud. This delivers an ultimate flexibility to select an appropriate communication method that suits the requirements of the given health application. As a result, the fog nodes and eHealth devices can be connected to the cloud using any hardware over any communication channel (wired networks, wireless networks, 3G/4G, or even satellite) based on a wide-range of protocols (MQTT, WebSocket, REST API, etc.).

- User, device and data management: the cloud integrates data from multiple sources. It captures the data from many fog nodes and stores the data safe and secure. So it is always there to be accessed by those engaged in the patients care. This platform is also integrated seamlessly with non-sensor sources such as data from EHRs, e-prescription platform, web sources, etc. Therefore, patient, physicians or anyone else in the patient's care team can access the data anytime and anywhere they need to. This significantly increases the collaboration across all disciplines increasing the efficiency of healthcare plan. Another advantage of the cloud platform is that it separates the data layer from the application layer while providing a unified schema in terms of capture and query transactions. This feature results in more flexibility to develop new applications. This module includes built-in capabilities for managing users, groups, devices and fog nodes, access permissions and roles.

- eHealth application service [19]:

- Dashboard: a web-based application that allows patients and those engaged in the patients care to monitor and manage the connected eHealth devices, and the aggregated medical big data. The dashboard enables the users to remotely monitor, configure, control, diagnose, and repair the eHealth devices connected to the cloud. It also allows the users to view, visualize, understand, and share their data.

- Rule engine: a holistic engine with a web-based UI for analyzing the incoming data to create appropriate events, alarms, notifications, and trigger actions. The rules can be as simple as a threshold to complex rules consisting of several analytical expressions and scheduling.

- Big data analytics: it is very important to analyze the aggregated medical data to effectively identify actionable insights. Several event processing, rule-based processing, data mining and machine learning, and also automated reasoning based algorithms can be applied on the stored historical records to extract meaningful insights about patients' health. For example, in predicting the onset of a disease, the patient can take action early in order to significantly improve the outcome, while reduce the costs. The extracted insights also enable the users to update the rules and the configurations of the connected eHealth devices and the fog nodes for process changes and optimizations. For instance, it can adjust the sampling rate of data, and the resolution multimedia (images and videos). 
- Integration framework: a holistic scalable message queue technology framework that facilitates cloud-to-cloud communication and integration with any system, application or portal. The framework also delivers a set of rich REST API with strong reliable authentication and authorization features providing access to the logic and data tiers of the eHealth cloud.

\section{IOT EHEALTh SERVices AND APPLiCATIONS}

In this section, we briefly discuss some of the applications of the proposed IoT eHealth ecosystem and architecture.

\section{A. mHealth}

Thanks to the cloud platform, whether patients are traveling on the road or relaxing at home, the health information always stays with them, and they can access it using mHealth smartphone apps or web-based cloud dashboard. The care givers can also leverage the platform and mHealth smartphone apps with $\mathrm{P} 2 \mathrm{P}$ video/audio capabilities to help and guide patients at anytime, and anywhere. Patients conveniently get diagnosis, treatment, as well as prescription refills from care givers whenever they require it. Considering the fact that health professionals have access to the holistic health database of patients on the cloud platform 24/7, patients receive the most accurate treatment possible. Therefore, the proposed holistic IoT eHealth ecosystem ensures that patients will always receive the best care available [19].

\section{B. IoT in Ambient Assisted Living}

The proposed IoT eHealth ecosystem allows the incapacitated and aging individuals to live longer and healthier. It is observed that the share of the aging population is significantly increasing and it is estimated that about $20 \%$ of the world population will be over 60 years old by 2050 [98]. At the same time, aging brings fast growth of various chronic diseases (e.g., stroke, cancer, type II diabetes, and obesity). Thanks to the growing acceptance of technology, in Abient Assitet Living (AAL), IoT enables indoor positioning as well as location-aware real-time monitoring of living parameters (e.g., heart rate) and environmental conditions [64], [99], [100], [28].

\section{IoT Medication}

IoT can also enable us to detect adherence to medication and to prevent fatal Adverse Drugs Reaction (ADR) [87], [101]. As studied in [87], combination of smart pill bottle technologies, wearable audio sensors, and classification techniques is capable of assessment of medication adherence with high accuracy. As stated in [101], ADR rate is about 6.5\% in worldwide hospitals. IoT medication with help of NFC-enabled smart pill bottle, cloud-based Electronic Health Record (EHR), and a knowledge-based system can significantly prevent the adverse consequences of wrong drug usage.

\section{IoT for Individuals with Disabilities and Special Needs}

In 2011, WHO conducted its first survey on disability and reported that more than a billion people (equivalent to $15 \%$ population of world) live with disability [102]. IoT eHealth can bring a great deal of comfort to this vulnerable population and enhance their life significantly through automated, timely, reliable resistive technologies. For example, a number of smart gloves are developed with low-cost inertia sensors enabling hearing loss to communicate with those who are not very familiar with the American Sign Language (ASL) [103], [104], [105], [102]. Smartwatches are being used for patients with speech disorders to train their speech functions in remote settings [106], [93]. IoT systems are built in smart cities for improving the access for wheelchair users who face mobility related challenges in everyday life [107]. IoT also can help gather information from individuals about their special needs remotely in their comfortable environments [108]. Schools can leverage IoT platforms to make special needs education more efficient and accessible to the children with disabilities. The Wireless Nano Retina Eyeglasses is a kind of IoT device that is already out in the market, allowing to communicate with retina implants in blind individuals for real-time fine-tuning of the visuals [109].

\section{E. IoT for Smart Medical Implants}

Beyond wearable devices, IoT eHealth brings new promises to implantable medical devices that are highly sophisticated, miniaturized, reliable systems inserted inside the body to restore or enhance the human functions [110]. Some of the examples of electronics implants are: 1) pacemakers that stimulate the heart muscle to help regulating its rhythm [111], 2) deep brain stimulation (DBS) systems that are also known as brain pacemakers provide highly-controlled electrical impulses into deep brain regions to reduce movements symptoms in motor disorders such as Parkinson's disease [112] and Essential Tremors [113], and 3) cochlea implants that stimulate electrodes placed inside the inner-ear to store hearing functions [114]. Such electronics implants have miniaturized circuits including an analog front-end, a micro-controller, and a battery-based power management module. The paradigm of IoT has been explored in the area of medical implants to make them more contextual, power-efficient, and secure. For example, there are ongoing research efforts in optimizing deep brain simulations through limb-worn inertia sensors [114], [115], [116]. IoT provides a basic framework for tele-management and programming of cochlea implants that are non-trivial tasks for patients who generally have to travel to implant centers for programming services [117]. 


\section{F. IoT-based Early Warning Score (EWS)}

Due to large volume of incoming medical data generated by a wide-range of bio-sensors and hundreds of thousands of patients, it is infeasible to monitor every patient directly. To assist the health professionals, an IoT-aware Early Warning Score System (EWS) can be utilized to effectively detect and forecast deterioration of patients' conditions early in time [90], [91]. The basic idea behind EWS is to process and analyze six cardinal vital signs including temperature, respiratory rate, systolic blood pressure, pulse rate, oxygen saturation, and level of consciousness. The measured vital signs are then mapped to a composite patient deterioration risk score. Indeed, each vital sign is processed and a score is assigned to it in such a way that the magnitude of the score represents the deviation of the parameter form its corresponding norm. Combining all the scores leads to a composite reflecting the overall deterioration risk of the patients. Note that EWS is an established approach which is widely used by many hospitals across the world. Several studies have shown that EWS can predict complications of patients about 24 hours in advance [91]. In this context, the proposed IoT eHealth ecosystem enables caregivers to continuously collect vital signs remotely and compute the deterioration risk of patients entirely in an automatic way remotely. This revolutionary approach potentially is capable of identifications of deteriorating patients early in time, and hence it can save lives and reduce mortality of patients.

\section{G. IoT-based Anomaly Detection}

EWS has two shortcomings. First, it does not consider full spectrum of bio-signals such as Cutaneous water/sweat. Moreover, it is developed based on supervised machine learning techniques, and thus it might not be able to effectively capture those abnormalities that are not within the knowledge of the supervisor [118]. Indeed, an accurate anomaly detection system requires to learn continuously over time. To overcome these critical issues, we propose to use an accurate anomaly detection system that learns and evolves continuously over time. This system is based on Hierarchical Temporal Memory (HTM), a biologically inspired machine learning unsupervised intelligence technology [119]. Thanks to this technique, health professionals can quickly identify temporal anomalies which might be a sign of severe problems such as heart attack, and stroke. Fig. 8 shows the key steps that the system uses to detect anomalies. Time series bio-signals captured by connected sensors and IoT eHealth devices are forwarded to fog nodes. Fog nodes filter, process, extract features, and compress the raw data. Next, the processed data and its corresponding time stamp are transferred to the cloud using a secure connection. Then, an encoder converts the received data to a Sparse Distributed Representation (SDR). The generated SDRs are fed into the HTM machine learning module. HTM indeed tries to mimic the behavior of neocortex of brain by learning the temporal patterns of SDRs continuously. Over time, HTM generates an adaptive sophisticated model to predict the next incoming sequence of SDRs. If the incoming sequence of SDRs does not match with what predicted, an anomaly alarm is triggered. In order to reduce the false positives mainly due to variations in data and/or noise, HTM computes a time-varying average of the error and compares it with the distribution of errors. Based on this comparison, HTM can also estimate the accuracy of the predicted anomaly and warning. Finally, the outputs of the risk analysis and warnings can be visualized in the dashboard and also they can be directed to patients and those engaged in the patients care.

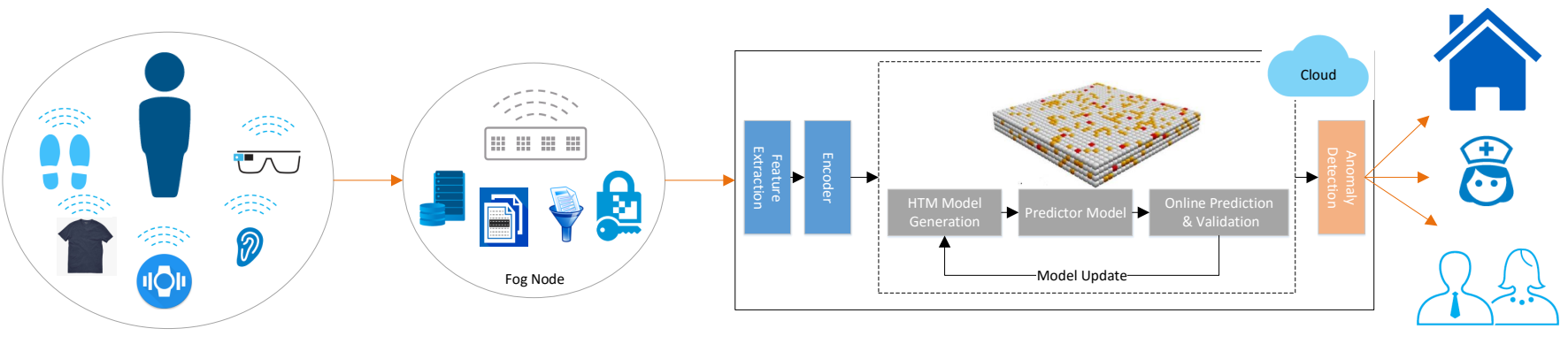

Fig. 8: IoT-based Anomaly Detection System.

\section{H. Population Health Management}

As estimated by IBM, medical data is expected to double every 73 days by 2020 [120]. Big data analytics can enable us to understand the medical data and extract deep insight in order to personalize the care plan, perform early interventions, improve the case and outcome, while reduce the healthcare cost. In this context, as reported by IBM [120], Medical Center of Columbia University applied big data analytics to examine medical data from patients who suffer from bleeding strokes in order to forecast the major complications 48 hours in advance compared to the traditional techniques [120]. Rizzoli Orthopedic Institute utilized big data analytics to understand the clinical variability within families with hereditary bone diseases. Their technique results up to 30 percent decrease in annual patient hospitalizations. The Hospital for Sick Children studied different 
big data analytic methods to process several vital signs of patients in order to predict hospital infection up to 24 hours in advance than traditional methods. In addition, several machine learning techniques have been proposed automatically to detect psycho-physiological stress from bio-signals such as accelerometer and skin conductance [121], [122]. The authors of [118], constructed a behavior model based on time series data using machine learning techniques for fast detection of abnormality. In general big data analytics can answer the following critical questions:

- How to perform an alarm-based screening by predicting which individuals are at most-risk?

- Which care plan and treatment results in the best outcome?

- How to forecast complications by processing bio-signals and medical data?

- How to create an automatic diagnostic system (e.g., using classification techniques) to assist patients even without the presence of a healthcare professional?

- How to create a recommendation system (e.g. using collaborative filtering system) in order to recommend the most effective treatment and even HCC gene based on similarity measures between patients and/or treatments?

\section{IoT eHealth Challenges}

While IoT eHealth comes with the promises and visions of seamless connectivity across the physically distant locations where patients, clinics and hospitals could cooperate, coordinate and orchestrate the healthcare processes, there are several research challenges that IoT eHealth has to overcome before it could become a mainstream platform:

\section{A. Data Management}

Data management challenges for IoT eHealth is similar to those faced by IoT in other domains. However, the eHealth data come from medical sensors attached to humans. The human body is a dynamic system that changes its state continuously. Hence, as seen in IoT eHealth applications, there will be a constant flux of data coming from edge sensors via fog computing nodes. The cost of sensors and computing is declining and hence, it has become cheaper to collect the big data in a short time. In other worlds, IoT eHealth has to handle the complexity of the data in terms of their variety, volume and velocity [123], [124], [125]. The challenge of data variety in IoT is quite newer than what existed 10 years ago. There are dozens of data formats depending on the healthcare end-user applications. For example, ECG data could be communicated in XML format, while detecting skin diseases using camera-based IoT device need to handle image formats [126]. The data format support for edge computers is dependent on the manufacturers and their target customers. In addition to edge data format, the data model on the cloud also varies and therefore, demands standardization. The challenges of data volume and velocity are more associated with the capabilities of fog node hardware to receive, process, store and communicate the high-fidelity, high-resolution data coming from medical devices that could be with patients or in hospitals or clinics. Therefore, there will be a need of fog admins who could oversee the data flux between the fog and cloud computing.

\section{B. Scalability}

To build a smaller scale of IoT, sensors on portable devices for data collection and secure central servers for processing users' requests are used to ensure all users can directly access medical services via portable devices such as smartphones. This facility can be scaled up to the entire hospital, so that patients in the hospital can use medical services, check updates and health status monitoring by their smartphones. This eHealth model can be scaled up to the entire city, if there are sensors and antenna in the city to collect data, smart big data algorithms and APIs to process data and analyze users' requests, and intelligent interfaces to inform the status of users' requests in real-time. In an eHealth-aware smart city, all data can be collected, processed and analyzed by smartphones through mobile apps and feedback will be sent seamlessly to patients to allow them to know their health status and results of their medical checks. When patients use medical services in IoT, it can save their time to wait for appointments, wait for results and have direct access to certain level of medical resources. Benefits of scalability to a smart city level can include improvement in efficiency, saving quality time for waiting and building direct relationship and trust between medical staff and patients [127].

\section{Interoperability, Standardization and Regulatory Affairs}

In general, IoT has raised concerns in the area of standardization. All manufacturers, service providers, and end users seek standards for operability both within and between the domains targeted by IoT applications. The standardization complexity lies in the fact that IoT aims to capture a wide range of disciplines that are, in general, regulated by different regulatory affairs. In the case of IoT eHealth, the complexity even increases due to the strict regulations mandated by medical standards. For example, in USA the standardization of wireless medical devices demands a multi-agency regulatory environment [128], involving three agencies: i) Food and Drug Administration (FDA), ii) Centers for Medicare and Medicaid Services (CMS), and iii) Federal Communications Commission (FCC). This implies that companies have to precisely consider the policies and rules mandated by all three agencies. Similarly, IoT eHealth will have to navigate through a complex multi-agency regulatory structure before we start to see the IoT eHealth products in the market. IoT eHealth will confront with similar trends in other parts of the world. 


\section{Interfaces and Human-Factors Engineering}

One of the key factors in IoT eHealth is the interface of the front-end technologies sensors, smartphones, tablets, computers, and other forms of interactions. When IoT medical devices are designed and given to the patients who have no experience of using high-tech tools, it becomes very important that end-users would be able to self-train themselves with these devices. In general, end-users have limited knowledge about the wireless networking, sensor syncing and other operations of the device. Moreover, when the devices will go in remote environments, setting up the eHealth systems will need to be straightforward and autonomous. For example, elderly population will be one of the largest stakeholders of IoT eHealth. Hence, device interfaces need to be user (patient)-friendly and to require minimum involvement of experts. One of the successful practices in humanfactors engineering is a participatory design [129], in which stakeholders or end-users of IoT eHealth devices could become a part of the design team to provide continuous feedback of their likes, dislikes, and comforts.

\section{E. Security and Privacy}

Every single IoT device might present a potential risk that could be exploited to either harm the end-users or jeopardize the privacy of them [130]. If IoT security is compromised, end-users information can be accessed through unauthorized authentications. Furthermore, this can lead to creating risks to personal safety. Security and privacy of IoT eHealth spans the whole lifecycle of the system starting from specification generation, to implementation and deployment [131], [132], [133], [134], [49], [135], [136], [137], [138]. However, protecting IoT eHealth ecosystem is a sophisticated and challenging task. In order to push IoT eHealth, these challenges must be overcome by taking a holistic multi-layer approach as discussed below:

1) Device layer: A few key examples of devices are connected sensors, medical devices, gateways, fog nodes, and mobile devices that capture, aggregate, process and transfer the medical data to the cloud. The most common attacks at device layer are tag cloning, spoofing, $R F$ jamming, cloud polling and direct connection. In cloud polling attack, attackers try to redirect the network traffic in order to inject their commands to the device [132], [139]. This can be achieved by several means such as Man-in-the-Middle (MITM) attack and modifications of the settings of domain name system (DNS). To tackle this attack, IoT devices must always evaluate and verify that the received certifications really belong to the eHealth cloud. In the direct connection attack, attackers can use Service Discovery Protocol such as Universal Plug and Play (SSDP/UPNP) protocol or built-in capabilities of BLE to find and discover IoT devices. To overcome/solve this type of attacks, unauthenticated requests should be ignored and blocked by IoT eHealth devices using robust cryptographic algorithms and key management system. In general, security measures at device layer include identity, authentication, and authorization management, secure booting (i.e., prevent unauthorized applications to be executed), application sandboxing, whitelisting, fine-grained access control capability of resources, protection of data during capture, storage, and transit, traffic filtering feature, fault tolerance, password enforcement policies, secure pairing protocols, and secure transmission mechanisms [139], [140]. Note that during implementation of security algorithms for IoT eHealth devices, the extremely limited resources of such devices (e.g. memory, processing capabilities, power resource, network range, embedded operating systems, thin embedded network protocol stack) should be carefully considered [135].

2) Network layer: This layer is responsible to establish appropriate connections between sensors, IoT eHealth devices, fog nodes and eHealth cloud based on an avalanche of mix network protocols (such as Wi-Fi, BLE, ZigBee). The most common attacks at this layer are Eavesdropping, Sybil attack, Sinkhole attack, Sleep Deprivation attack, and Man-in-the-Middle attack. To secure the network layer, it is very important to use trusted routing mechanisms, message integrity verification techniques (using hashing mechanisms such as MD5 and SHA) as well as point to point encryption techniques based on cryptographic algorithms. Cryptographic algorithms can be classified into two groups, namely symmetric algorithm (AES, DES, Blowfish, and Skipjack) and asymmetric or public-key algorithms (Rabins Scheme, NtruEncrypt and Elliptic Curve Cryptography). Note that symmetric algorithms are less compute-intensive, and hence they are more suitable for low-power 8-bit/16-bit IoT devices. However, they usually suffer from key exchange mechanisms and key confidentiality problems [141].

3) Cloud layer: The primary techniques to tackle security issues of cloud applications have been widely investigated in literature. However, any company that deliver eHealth products/services must take an efficient and adequate mechanism to combat the adverse impact of attacks. Denial-of-service (DoS) attack, SQL injection, malicious code injection, Spear-Phishing attack, sniffing attack, path traversal, unrestricted file uploading (remote code execution), cross-site scripting (XSS), Trojan horses, viruses, and brute-force attack (using weak password recovery methods) are among the top common cloud vulnerabilities [139].

4) Human layer: The basic, while important concept of IoT eHealth security is to give training to individuals to prevent disclosure of their critical medical data. For example, if skilled attackers get physical access to an individual's IoT eHealth device, they can read the corresponding internal memory/firmware, and modify the configuration settings in order to fully/partially control the device. Other typical security mistakes among users in eHealth domain are selecting weak passwords (such as 1234), purchasing used medical equipments, and sharing physical/electronic keys. 


\section{CASE Study}

The following case studies provide illustrative examples on how data may be collected nonobtrusively for telemedicine. Each case study has a different methodolgy for collecting, processing, and transporting data. This shows off the ability, to "plug and play" different protocols for further research on the most appropriate protocols for reducing overall response times.

\section{A. Smart Eyeglasses}

In case study, we use a smart eyeglasses for unobtrusive and continuous Heart Rate (HR) monitoring as our IoT eHealth device [63]. This device consists of an amped pulse sensor with green LED light [142], a ATmega32U4 micro-controller, a 3.7V/300mAh, Lithium Ion rechargeable battery, and a Nordic nRF8001 BLE module. The micro-controller processes the amplified output signals of the sensor to determine the HR. Then it sends the HR data samples via BLE to the fog node. Fig. 9 shows the functionality of our IoT eHealth device.
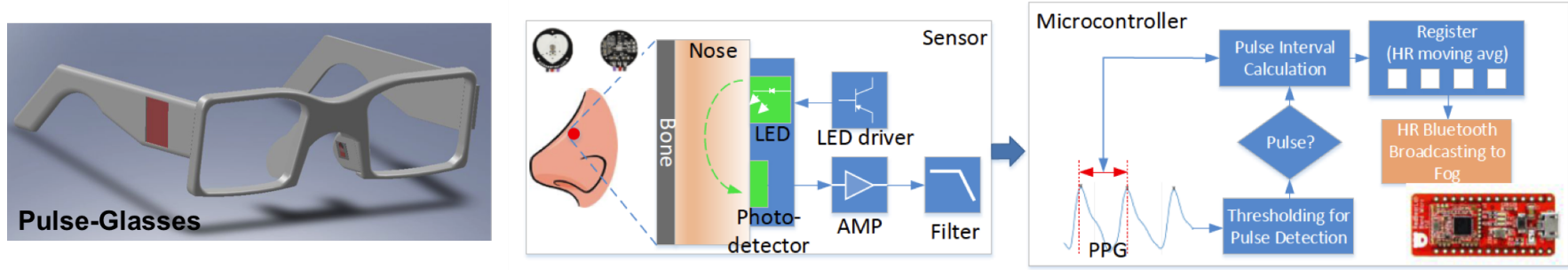

Fig. 9: Hardware architecture of IoT eHealth device: Pulse-Glasses.

The fog node is responsible to aggregate HR values from smart glasses, convert them into XML format, watermark the XML file based on a technique presented in [143], and upload them to the eHealth cloud based on MQTT protocol. The fog node is designed based on Qualcomm Snapdragon 410 Quad-core Processor. The processor is based on ARM Cortex A53. In this processor, the frequency of each core can be increased up to $1.2 \mathrm{GHz}$. The board is equipped with Wi-Fi $802.11 \mathrm{~b} / \mathrm{g} / \mathrm{n}$ 2.4GHz, and Bluetooth 4.1 connectivity and it also has 1GB LPDDR3 533MHz/ 8GB eMMC 4.5/SD 3.0. Moreover, the board includes Qualcomm Adreno 306 GPU and several connectors for HDMI, UART, SPI, I2S, I2C, GPIO x12, USB, I2C, 2L+4L MIPI-CSI. The operating system of the board is Linux based on Debian 8.0.

Our eHealth cloud is developed based on Redis as in-memory data structure store and caching system [144], Kafka as our distributed, publish-subscribe messaging system [145], MongoDB as our main database [146], and Hadoop as the storage of our big data [147]. MQTT broker is implemented by Node.js transporting the data into the MongoDB and Redis finally connected to Kafka by a bridge for scalability. In addition, our big data analytic is done using GraphLab, a fast, scalable machine learning platform [148]. The main functionalities of the cloud are i) providing a dashboard (See Fig. ??), and ii) providing an early warning system based on HR. This holistic HR monitoring system is validated in hospital trials using 100 patients (both male and female in the range of 20-70 years old) with a cutting-edge ECG system. Fig. 11 shows the results of our system for a patient (22 years old male) in three different conditions [63].

\section{B. Smart Glove with Fog-Driven IoT}

In this study, we used a smart glove that is targeted to meet the requirements of telemedicine in Parkinson's disease [149]. The smart glove is stitched with flex sensors on the pointing finger and the thumb to detect the motor symptoms in the hand such as tremors, rigidity, and slowness of movements (see Fig. 12). The Smart Glove uses Arduino 101, hosting Intel Curie chip for on-board computation and Bluetooth low energy for communication with local network. The Curie Chip reads the voltage across the flex sensors and converts it to the resistance of them. We adopted the finger tapping motor task that is one of the exams in the Unified Parkinson's Disease Rating Scale (UPDRS) [150]. One of the key features observed by neurologists is the frequency of tapping. For example, neurologists measure the rate of variation on tapping speed and record a clinical score for this task. The finger tapping task is particularly chosen since it has a higher clinical value and is easy for patients to perform in home settings.

1) Data Collection and Signal Processing: The task requires the patients to tap pointing finger and thumb for 10 times. Therefore, our study included a healthy participant conducting five rounds of finger-tapping task. The participant was asked to vary the rate of finger tapping frequency in each round. We developed a peak detection algorithm to measure the intensity of the tapping at each instance. We applied amplitude threshold to detect the peak. If the amplitude of the signal is greater than a threshold and is bigger than both previous and next samples, we consider it as a peak. We also implemented a temporal threshold, because each pinch has a few micro movements or small fluctuations that result in more than one peak at the time. Therefore, we make a temporal filtration such that if the time interval between two peaks is too small (in this case it is set to 


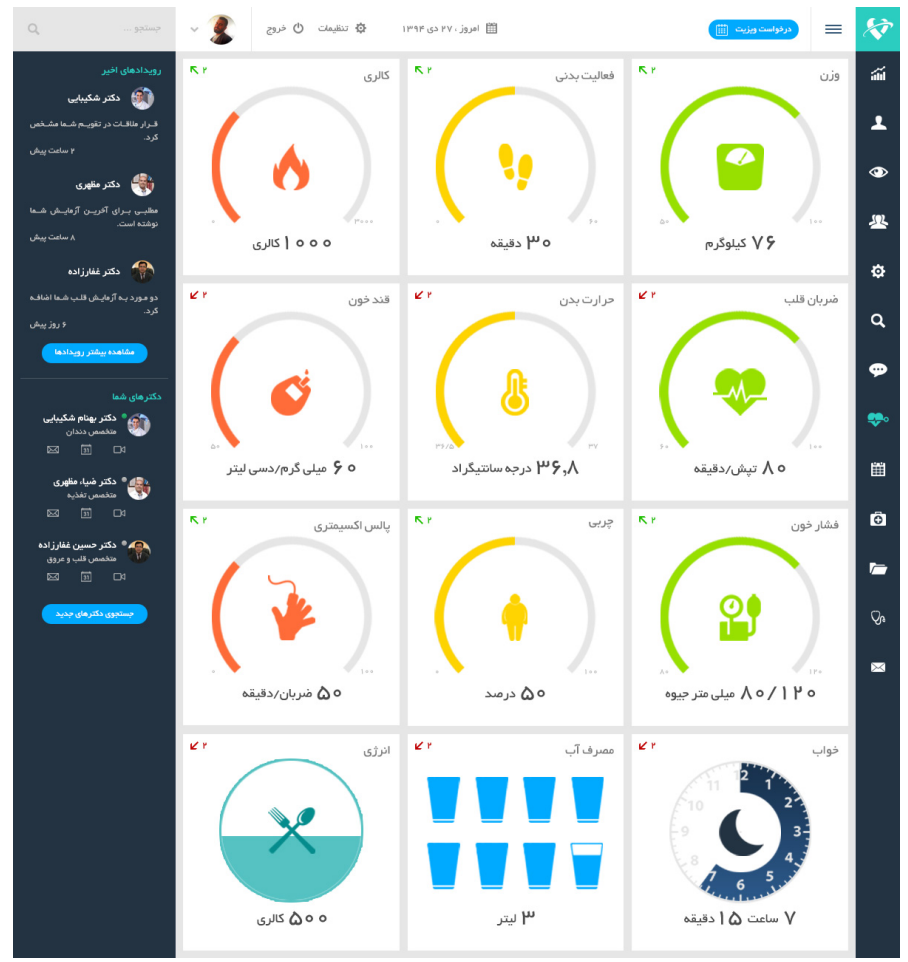

Fig. 10: A screenshot of the Patient Dashboard
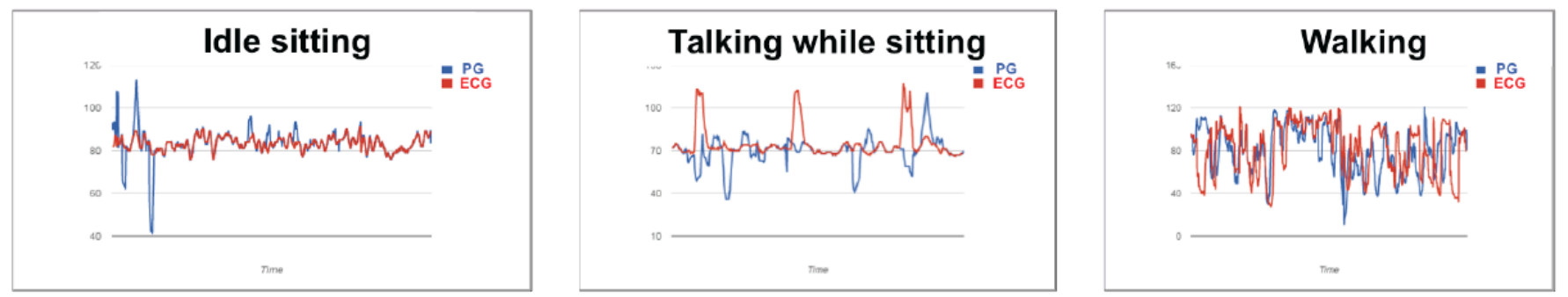

Fig. 11: Preliminary HR monitoring using Pulse Glasses. Each graph plots 10min HR data.

be less than 1/3 of a second), we do not consider them as different peaks for different pinches. The peaks are shown in Fig. 13. After finding the peaks, we calculate the time between each two adjacent peaks and convert it to a frequency (shown in Fig. 14). We can see that the frequency of pinching increases when the subject is doing it faster.

2) Smart Gloves in Fog-Driven IoT Environment: As shown in Fig. 15, we have designed a fog computing environment for low-power embedded processors based on [60], [151], [152]. We conducted a benchmark experiment using Intel Edison and Raspberry Pi (version 3) that serve as an embedded fog computer to run an i386 Debian/Jessie distribution giving us the ability to run Octave 3.8.2-4 for processing of the data. The data collected from the Smart Glove is stored into a text file. The embedded fog computer (Edison or Raspberry Pi) acts as a gateway to the cloud as well as a platform for processing the data. The Edison platform is designed with a core system processing consisting of dual-core, dual-threaded Intel Atom CPU at $500 \mathrm{MHz}$ and a 32-bit Intel Quark microcontroller at $100 \mathrm{MHz}$, along with connectivity interfaces capable of Bluetooth 4.0 and dual-band IEEE $802.11 \mathrm{a} / \mathrm{b} / \mathrm{g} / \mathrm{n}$ via an onboard chip antenna. The Raspberry Pi platform has a $900 \mathrm{MHz} 32$-bit quad-core ARM Cortex-A7 CPU, and 1GB RAM.

We developed a benchmarking process for timing, CPU load, and memory load measurements. The results were generated by combining the information collected from the Octave Function Profile, and the Linux program top. The Profile function running inside Octave collected timing specifics for out particular algorithm, while the top program collected timing, CPU and memory loads for the entire process. This means that the measurements shown in the total process breakdown in the figure below, includes the load added on by starting an instance of Octave. We observed that the run time for an Edison (4s) was much greater than that of the Raspberry Pi $(2.5 \mathrm{~s})$, and that an increase in data sets $(\mathrm{N})$ produced a processing time of the order 


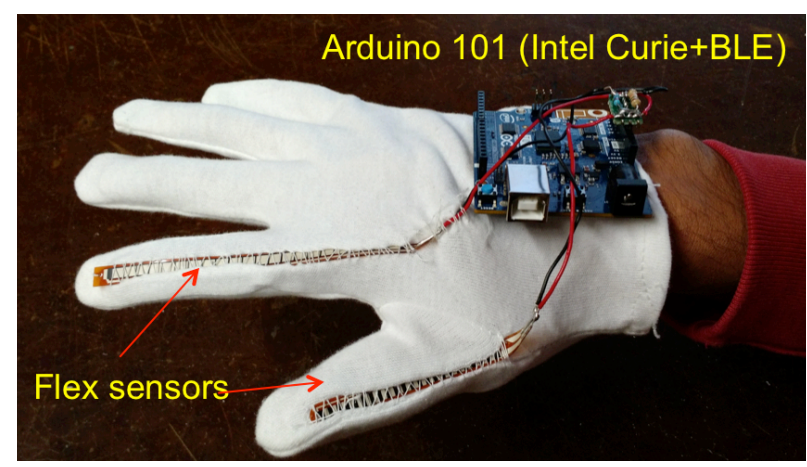

Fig. 12: The Smart Gloves.
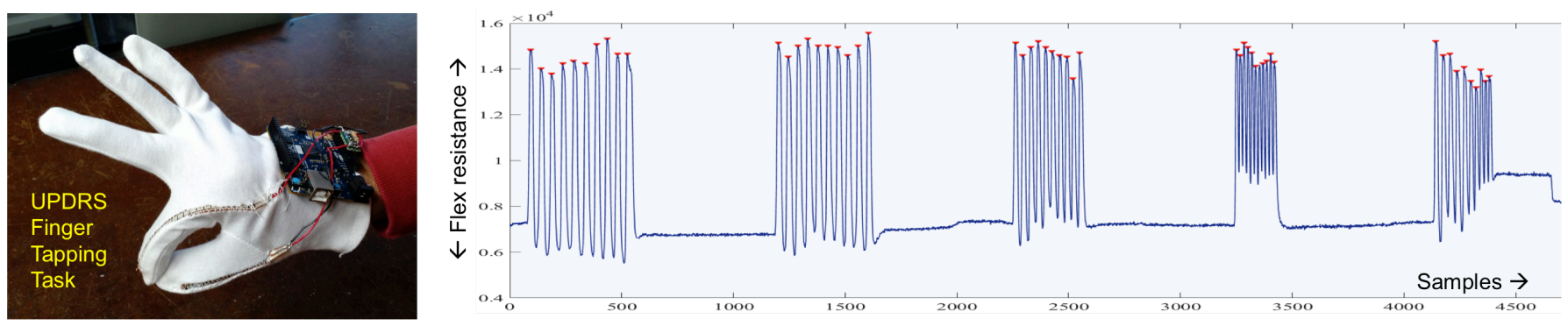

Fig. 13: The data collection and signal processing of the UPDRS finger tapping task. A healthy participant performed five rounds of the task.

$\mathrm{N} \log (\mathrm{N})$. It should be noticed that the Raspberry Pi could complete the process almost $2 \mathrm{x}$ faster. Furthermore, it could scale to $125+$ datasets while the Edison would gracefully crash (see Fig. 16).

The network we created places the fog node (in our case the Edison and Raspberry Pi) in between the Smart Glove and the cloud. Its assumed that the arrival and service processes are geometrically distributed. Since the fog node will be placed in locations with only a small number of devices in the area, such as a nursing home, it is assumed that the mean arrival rate for data will be once per minute. The service rate for the fog nodes can be surmised from Fig. 16 under total runtime. Using Littles Law we can determine the average wait time for each device. When running one set at a time, we found that the average wait time for the Edison and Raspberry Pi will be roughly 64.65 seconds and 12.39 seconds, respectively.

Each of these fog nodes are used to collect data from the Smart glove, process the data received, and finally post the processed data to a server in the cloud. Fig. 17 shows a break down in the average amount of time spent for each step. The Raspberry Pi provided a service rate that is just a third of the Edisons. Furthermore, in active mode, the Raspberry Pi consumes $198 \mathrm{~mW} / \mathrm{s}$ and the Edison consumes 529mW/s (see Fig. 17).

\section{CONCLUSiOnS}

There is an increasing need from clinic-centric healthcare to patient-centric healthcare. IoT is expected to be a strong enabler by providing a seamless connection of devices and cloud storage as well as acting agents such as patient, hospital, analysis labs, and emergency services. A typical IoT eHealth system consists of four layers: 1) sensing layer, which integrates with all different types of hardware connect to the physical world and collect data, 2) networking layer, which offers networking support and data transfers in the wired and wireless networks, 3) service layer that creates and manages all types of services aiming to satisfy user requirements. 4) interface layer, which offers interaction methods to users and other applications.

Due to the vast amount of different applications with different quality-of-service, storage and latency requirements, we observe a tendency to split the networking layer into two sub-layers: fog and cloud layers. Fog layer handles the local buffering and different connectivity requirements to the device. Cloud layer handles the connectivity to fog, user/device/data management, and application services covering dashboard, rule engine, big data analytics, and integration framework within virtually any system, application or portal. We mostly observe that most of the work was done so far on the device sub-layer as part of sensing layer with examples in smart wearable devices, monitoring fitness devices, and medical-grade devices used in the hospitals but less work on fog and cloud sub-layers.

In this paper we presented a complete survey of various published papers on IoT eHealth and proposed a holistic eHealth ecosystem covering those layers where various applications can be mapped to those layers, which include mobile health, assisted 


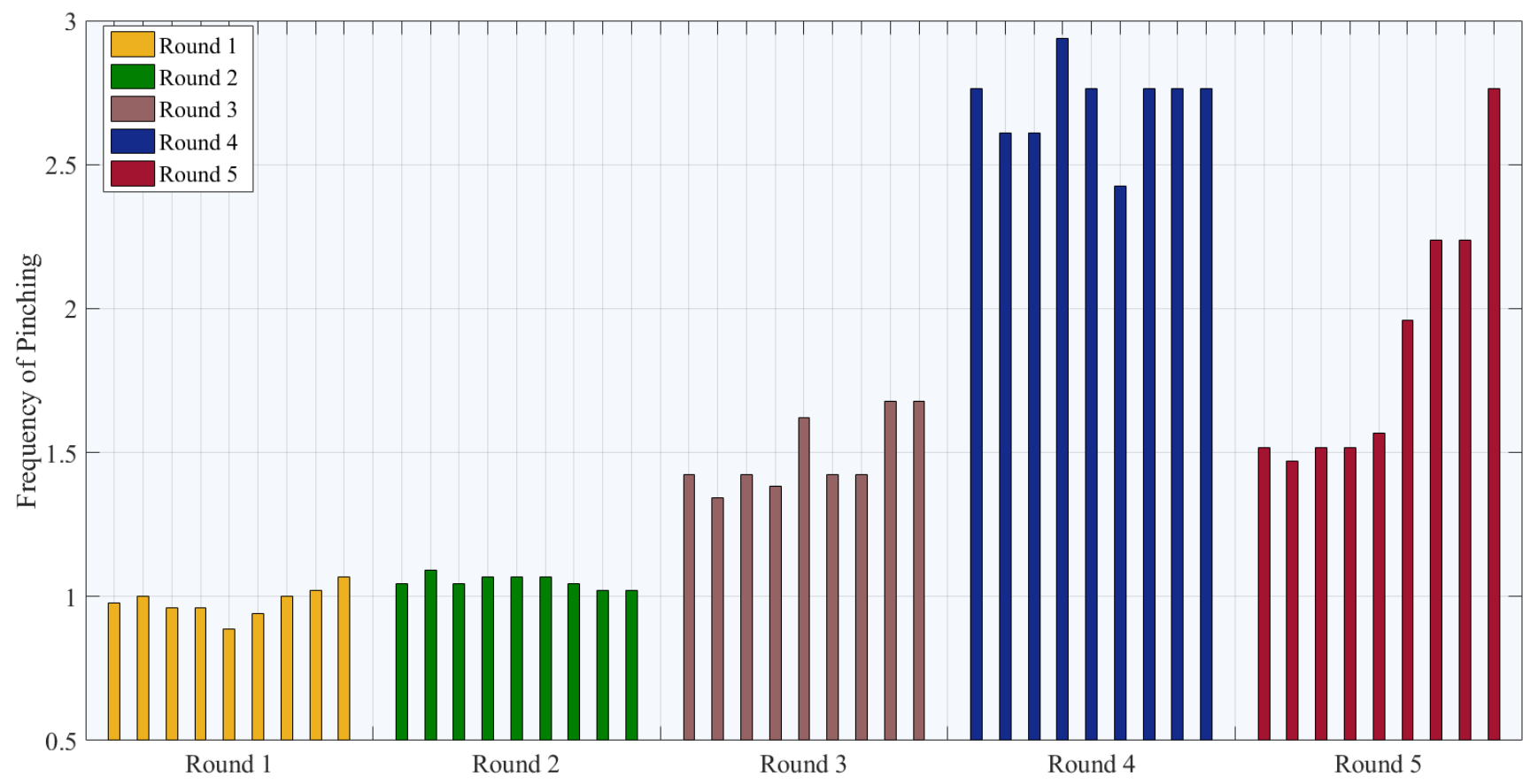

Fig. 14: Frequency of tapping/pinching measured by The Smart Glove.
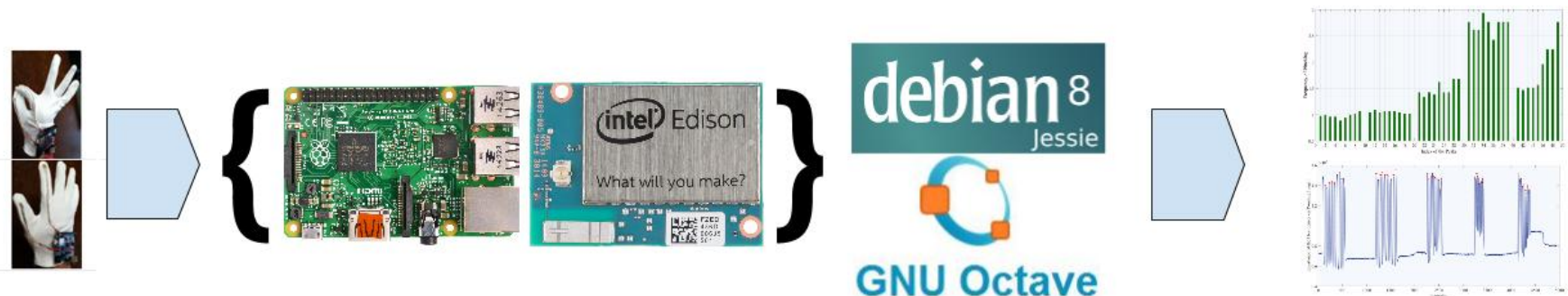

Glove Data

Sent to Raspberry Pi or Intel Edison

Processed with Octave on Debian

Data sent to cloud

Fig. 15: A benchmarking environment for measuring the performance of low-power embedded fog computers that compute the frequency of finger tapping.

living, e-medicine and implants, early warning systems, and population monitoring. Despite the advantages offered by IoT eHealth, many challenges need to be tackled. Those include data management, scalability, interoperability, device-networkhuman interfaces, security, and privacy. We see that data is becoming more complex in terms of its variety, volume and velocity. Network infrastructure needs to be scalable with increasing number of users, lower latencies, and higher bandwidths. Standardization needs to enable interoperability of different devices and data. Interfaces need to be simple and genuine enough making them accessible for everybody from children to elder people. More importantly but not least the security of device, network, cloud, as well as acting agents have to be enhanced by secure hardware, network protocols, cloud less vulnerable to attacks, and adequate training of people that handle the secure data.

\section{ACKNOWLEDGMENT}

We are greatly thankful for Mohammadreza Abtahi for his research work on the Smart Glove.

\section{REFERENCES}

[1] J. Gubbi, R. Buyya, S. Marusic, and M. Palaniswami, "Internet of things (iot): A vision, architectural elements, and future directions," Future Generation Computer Systems, vol. 29, no. 7, pp. 1645-1660, 2013.

[2] L. Atzori, A. Iera, and G. Morabito, "The internet of things: A survey," Computer networks, vol. 54, no. 15, pp. 2787-2805, 2010.

[3] A.-M. Vilamovska, E. Hatziandreu, H. R. Schindler, C. van Oranje-Nassau, H. de Vries, and J. Krapels, "Study on the requirements and options for rfid application in healthcare," 2009.

[4] H. Alemdar and C. Ersoy, "Wireless sensor networks for healthcare: A survey," Computer Networks, vol. 54, no. 15, pp. 2688-2710, 2010. 


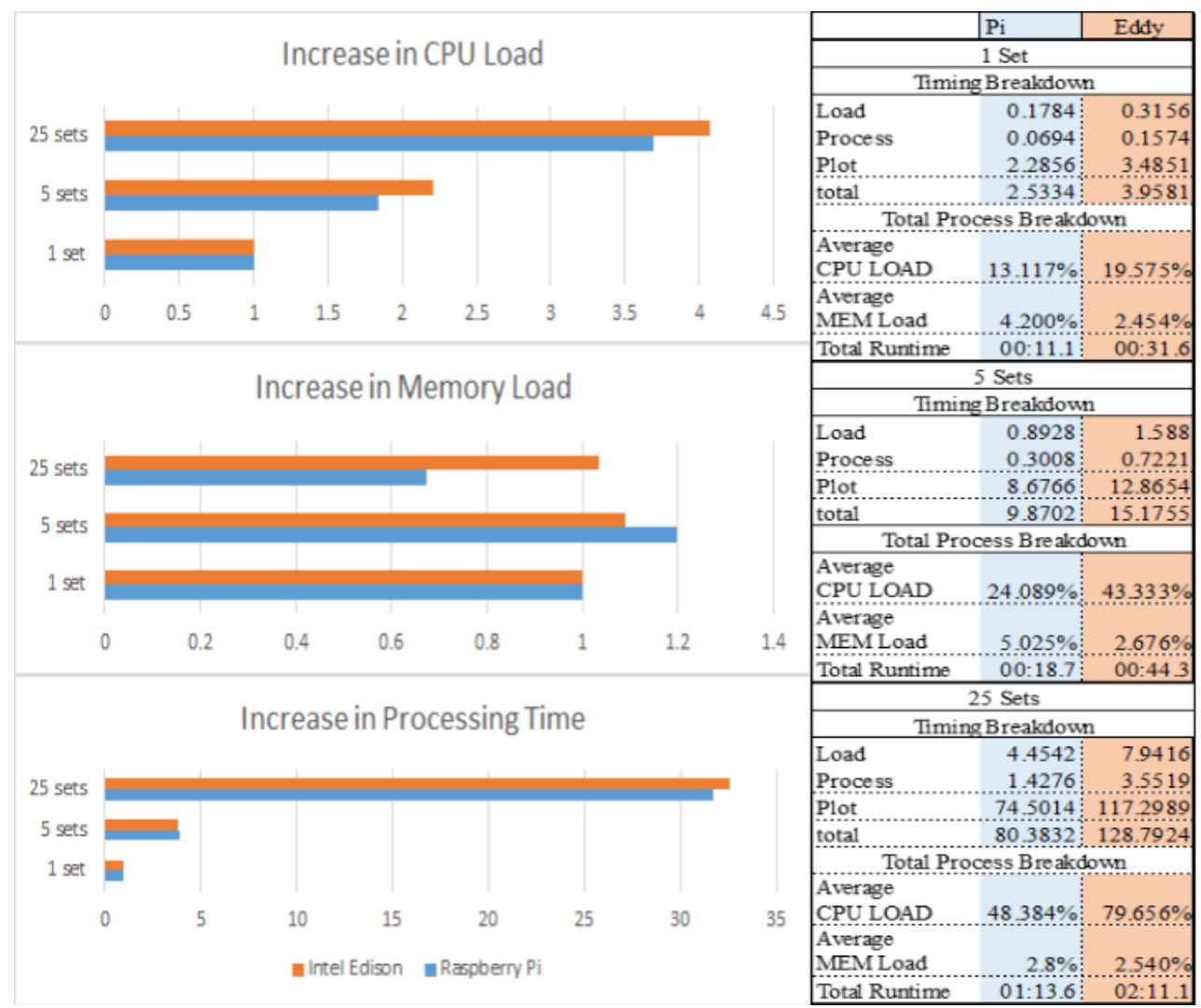

Fig. 16: the computing performance of Intel Edison and Raspberry Pi for 1, 5, and 25 sets of finger tapping data collected from the Smart Glove.

\begin{tabular}{|c|c|c|}
\hline \multicolumn{3}{|c|}{ Average Timing (s) } \\
\hline & Raspberry Pi & Intel Edison \\
\hline Receiving & 0.2 & 0.2 \\
\hline Posting & 0.2 & 0.2 \\
\hline Processing & 11.1 & 31.6 \\
\hline \multicolumn{3}{|c|}{ Average Power Consumption (W) } \\
\hline & Raspberry Pi & Intel Edison \\
\hline idle & 0.12567 & 0.1693 \\
\hline transmitting & 0.413 & 0.64124 \\
\hline processing & 0.1829 & 0.52072 \\
\hline total & 2.19539 & 16.711248 \\
\hline
\end{tabular}

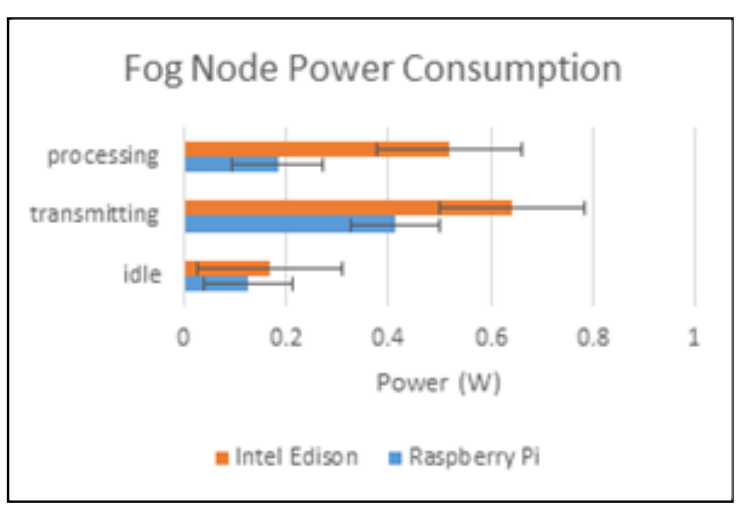

Fig. 17: Power consumption of fog nodes.

[5] B. J. Farahani and M. Fathy, "Improving coverage in mobile sensor networks using overlap concept," in Computer Conference, 2009. CSICC 2009. 14th International CSI, Oct 2009, pp. 633-639.

[6] B. J. Farahani, H. Ghaffarian, and M. Fathy, "A fuzzy based priority approach in mobile sensor network coverage," 2010.

[7] R. Soleimanzadeh, B. J. Farahani, and M. Fathy, "Pso based deployment algorithms in hybrid sensor networks," 2010.

[8] R. Soleimanzadeh, B. J. Farahani, M. Fathy et al., "Improved dynamic k-coverage algorithms in mobile sensor networks," Wireless Sensor Network, vol. 2, no. 10, p. 784, 2010.

[9] M. Saravi and B. J. Farahani, "Distance constrained deployment (dcd) algorithm in mobile sensor networks," in 2009 Third International Conference on Next Generation Mobile Applications, Services and Technologies, Sept 2009, pp. 435-440.

[10] L. Da Xu, W. He, and S. Li, "Internet of things in industries: a survey," Industrial Informatics, IEEE Transactions on, vol. 10, no. 4, pp. 2233-2243, 2014.

[11] E. Welbourne, L. Battle, G. Cole, K. Gould, K. Rector, S. Raymer, M. Balazinska, and G. Borriello, "Building the internet of things using rfid: the 
rfid ecosystem experience," Internet Computing, IEEE, vol. 13, no. 3, pp. 48-55, 2009.

[12] D. Miorandi, S. Sicari, F. De Pellegrini, and I. Chlamtac, "Internet of things: Vision, applications and research challenges," Ad Hoc Networks, vol. 10, no. 7, pp. 1497-1516, 2012.

[13] D. Bandyopadhyay and J. Sen, "Internet of things: Applications and challenges in technology and standardization," Wireless Personal Communications, vol. 58, no. 1, pp. 49-69, 2011.

[14] H. Sundmaeker, P. Guillemin, P. Friess, and S. Woelffle, "Vision and challenges for realising the internet of things. european commission information society and media," 2010.

[15] S. Haller, S. Karnouskos, and C. Schroth, The internet of things in an enterprise context. Springer, 2008.

[16] Z. Zheng, J. Zhu, and M. R. Lyu, "Service-generated big data and big data-as-a-service: an overview," in Big Data (BigData Congress), 2013 IEEE International Congress on. IEEE, 2013, pp. 403-410.

[17] D. A. Keim, J. Kohlhammer, G. Ellis, and F. Mansmann, Mastering the information age-solving problems with visual analytics. Florian Mansmann, 2010.

[18] V. Chang, Y.-H. Kuo, and M. Ramachandran, "Cloud computing adoption framework: A security framework for business clouds," Future Generation Computer Systems, vol. 57, pp. 24-41, 2016.

[19] Pirouzan Group, available at http://pirouzansystem.com/.

[20] D. Uckelmann, M. Harrison, and F. Michahelles, An architectural approach towards the future internet of things. Springer, 2011.

[21] M. C. Sokol, K. A. McGuigan, R. R. Verbrugge, and R. S. Epstein, "Impact of medication adherence on hospitalization risk and healthcare cost," Medical care, vol. 43, no. 6, pp. 521-530, 2005.

[22] D. o. E. United Nations and P. D. Social Affairs, "World population ageing," Computer Networks, 2013.

[23] "Bulletin of the world health organization," Computer Networks, vol. 88, no. 4, pp. 241-320, 2010.

[24] A. D. Association et al., "Economic costs of diabetes in the us in 2012. diabetes care 2013; 36: 1033-1046," Diabetes care, vol. 36, no. 6, p. 1797, 2013.

[25] J. M. Corrigan, E. K. Swift, M. P. Hurtado et al., Envisioning the national health care quality report. National Academies Press, 2001.

[26] C. F. Hill, B. W. Powers, S. H. Jain, J. Bennet, A. Vavasis, and N. E. Oriol, "Mobile health clinics in the era of reform." The American journal of managed care, vol. 20, no. 3, pp. 261-264, 2014.

[27] ntelligence M. Global Telemedicine MarketGrowth, Trends and Forecasts (2015-2019). 2015, available at http://www.mordorintelligence.com/industryreports/global-telemedicine-marketindustry.

[28] P. Rashidi and A. Mihailidis, "A survey on ambient-assisted living tools for older adults," Biomedical and Health Informatics, IEEE Journal of, vol. 17, no. 3, pp. 579-590, 2013.

[29] F. Sadri, "Ambient intelligence: A survey," ACM Computing Surveys (CSUR), vol. 43, no. 4, p. 36, 2011.

[30] E. Aarts and J. Encarnaçao, "The emergence of ambient intelligence," Berlin, Germany: Springer, 2006.

[31] M. Wang, G. Zhang, C. Zhang, J. Zhang, and C. Li, "An iot-based appliance control system for smart homes," in Intelligent Control and Information Processing (ICICIP), 2013 Fourth International Conference on. IEEE, 2013, pp. 744-747.

[32] H. Chourabi, T. Nam, S. Walker, J. R. Gil-Garcia, S. Mellouli, K. Nahon, T. A. Pardo, and H. J. Scholl, "Understanding smart cities: An integrative framework," in System Science (HICSS), 2012 45th Hawaii International Conference on. IEEE, 2012, pp. 2289-2297.

[33] C. Harrison, B. Eckman, R. Hamilton, P. Hartswick, J. Kalagnanam, J. Paraszczak, and P. Williams, "Foundations for smarter cities," IBM Journal of Research and Development, vol. 54, no. 4, pp. 1-16, 2010.

[34] Y. Sun, H. Song, A. J. Jara, and R. Bie, "Internet of things and big data analytics for smart and connected communities," IEEE Access, vol. 4, pp. 766-773, 2016.

[35] M. M. Rathore, A. Paul, A. Ahmad, and S. Rho, "Urban planning and building smart cities based on the internet of things using big data analytics," Computer Networks, 2016.

[36] N. S. Foundation, "Supporting research advances in smart and connected communities."

[37] M. A. M. Pla, L. G. Lemus-Zúñiga, J.-M. Montañana, J. Pons, and A. A. Garza, "A review of mobile apps for improving quality of life of asthmatic and people with allergies," in Innovation in Medicine and Healthcare 2015. Springer, 2016, pp. 51-64.

[38] J. Lambrechts and S. Sinha, "Tools and facilitators towards successful planning for sustainable cities," in Microsensing Networks for Sustainable Cities. Springer, 2016, pp. 269-305.

[39] S. Djahel, M. Salehie, I. Tal, and P. Jamshidi, "Adaptive traffic management for secure and efficient emergency services in smart cities," in Pervasive Computing and Communications Workshops (PERCOM Workshops), 2013 IEEE International Conference on. IEEE, 2013 , pp. 340-343.

[40] V. Stantchev, A. Barnawi, S. Ghulam, J. Schubert, and G. Tamm, "Smart items, fog and cloud computing as enablers of servitization in healthcare," Sensors \& Transducers, vol. 185, no. 2, p. 121, 2015.

[41] A.-M. Rahmani, N. K. Thanigaivelan, T. N. Gia, J. Granados, B. Negash, P. Liljeberg, and H. Tenhunen, "Smart e-health gateway: Bringing intelligence to internet-of-things based ubiquitous healthcare systems," in Consumer Communications and Networking Conference (CCNC), 2015 12th Annual IEEE. IEEE, 2015, pp. 826-834.

[42] J. Granados, A.-M. Rahmani, P. Nikander, P. Liljeberg, and H. Tenhunen, "Towards energy-efficient healthcare: An internet-of-things architecture using intelligent gateways," in Wireless Mobile Communication and Healthcare (Mobihealth), 2014 EAI 4th International Conference on. IEEE, 2014, pp. 279-282.

[43] Y. Li, L. Guo, and Y. Guo, "Enabling health monitoring as a service in the cloud," in Proceedings of the 2014 IEEE/ACM 7th International Conference on Utility and Cloud Computing. IEEE Computer Society, 2014, pp. 127-136.

[44] F. Fernandez and G. C. Pallis, "Opportunities and challenges of the internet of things for healthcare: Systems engineering perspective," in Wireless Mobile Communication and Healthcare (Mobihealth), 2014 EAI 4th International Conference on. IEEE, 2014, pp. 263-266.

[45] M. S. Hossain, "Cloud-supported cyber-physical localization framework for patients monitoring," 2015.

[46] J. Granados, A.-M. Rahmani, P. Nikander, P. Liljeberg, and H. Tenhunen, "Web-enabled intelligent gateways for ehealth internet-of-things," in Internet of Things. User-Centric IoT. Springer, 2014, pp. 248-254.

[47] F. Akhbar, V. Chang, Y. Yao, and V. M. Muñoz, "Outlook on moving of computing services towards the data sources," International Journal of Information Management, vol. 36, no. 4, pp. 645-652, 2016.

[48] M. S. Hossain and G. Muhammad, "Cloud-assisted industrial internet of things (iiot)-enabled framework for health monitoring," Computer Networks, vol. 101, pp. 192-202, 2016.

[49] S. Riazul Islam, D. Kwak, M. Humaun Kabir, M. Hossain, and K.-S. Kwak, "The internet of things for health care: a comprehensive survey," Access, IEEE, vol. 3, pp. 678-708, 2015.

[50] G. Yang, L. Xie, M. Mantysalo, X. Zhou, Z. Pang, L. Da Xu, S. Kao-Walter, Q. Chen, and L.-R. Zheng, "A health-iot platform based on the integration of intelligent packaging, unobtrusive bio-sensor, and intelligent medicine box," Industrial Informatics, IEEE Transactions on, vol. 10, no. 4, pp. 2180-2191, 2014. 
[51] M. F. A. Rasid, W. Musa, N. Kadir, A. M. Noor, F. Touati, W. Mehmood, L. Khriji, A. Al-Busaidi, and A. Ben Mnaouer, "Embedded gateway services for internet of things applications in ubiquitous healthcare," in Information and Communication Technology (ICoICT), 2014 2nd International Conference on. IEEE, 2014, pp. 145-148.

[52] L. Yang, Y. Ge, W. Li, W. Rao, and W. Shen, "A home mobile healthcare system for wheelchair users," in Computer Supported Cooperative Work in Design (CSCWD), Proceedings of the 2014 IEEE 18th International Conference on. IEEE, 2014, pp. 609-614.

[53] D. Bortolotti, M. Mangia, A. Bartolini, R. Rovatti, G. Setti, and L. Benini, "An ultra-low power dual-mode ecg monitor for healthcare and wellness," in 2015 Design, Automation \& Test in Europe Conference \& Exhibition (DATE). IEEE, 2015, pp. 1611-1616.

[54] P. Castillejo, J.-F. Martinez, J. Rodriguez-Molina, and A. Cuerva, "Integration of wearable devices in a wireless sensor network for an e-health application," Wireless Communications, IEEE, vol. 20, no. 4, pp. 38-49, 2013.

[55] "Realization of comprehensive detection algorithm of electrocardiogram signal at application layer electrocardiogram monitoring internet of thing," Jan. 19 2011, cN Patent App. CN 201,010,286,888. [Online]. Available: http://www.google.com/patents/CN101947112A?cl=en

[56] L. Hu, M. Qiu, J. Song, M. S. Hossain, and A. Ghoneim, "Software defined healthcare networks," Wireless Communications, IEEE, vol. 22, no. 6, pp. $67-75,2015$.

[57] F. Samie, L. Bauer, and J. Henkel, "An approximate compressor for wearable biomedical healthcare monitoring systems," in Proceedings of the 10th International Conference on Hardware/Software Codesign and System Synthesis. IEEE Press, 2015, pp. $133-142$.

[58] E. Svennberg, J. Engdahl, F. Al-Khalili, L. Friberg, V. Frykman, and M. Rosenqvist, "Mass screening for untreated atrial fibrillation the strokestop study," Circulation, vol. 131, no. 25, pp. 2176-2184, 2015.

[59] J. Engdahl, L. Andersson, M. Mirskaya, and M. Rosenqvist, "Stepwise screening of atrial fibrillation in a 75-year-old population implications for stroke prevention," Circulation, vol. 127, no. 8, pp. 930-937, 2013.

[60] H. Dubey, J. Yang, N. Constant, A. M. Amiri, Q. Yang, and K. Makodiya, "Fog data: Enhancing telehealth big data through fog computing," in Proceedings of the ASE BigData \& SocialInformatics 2015. ACM, 2015, p. 14.

[61] I. Chiuchisan, I. Chiuchisan, and M. Dimian, "Internet of things for e-health: An approach to medical applications," in Computational Intelligence for Multimedia Understanding (IWCIM), 2015 International Workshop on. IEEE, 2015, pp. 1-5.

[62] L. Fanucci, S. Saponara, T. Bacchillone, M. Donati, P. Barba, I. Sánchez-Tato, and C. Carmona, "Sensing devices and sensor signal processing for remote monitoring of vital signs in chf patients," Instrumentation and Measurement, IEEE Transactions on, vol. 62, no. 3, pp. 553-569, 2013.

[63] N. Constant, O. Douglas-Prawl, S. Johnson, and K. Mankodiya, "Pulse-glasses: An unobtrusive, wearable hr monitor with internet-of-things functionality," in Wearable and Implantable Body Sensor Networks (BSN), 2015 IEEE 12th International Conference on. IEEE, 2015 , pp. 1-5.

[64] R. Istepanian, S. Hu, N. Philip, and A. Sungoor, "The potential of internet of m-health things m-iot for non-invasive glucose level sensing," in Engineering in Medicine and Biology Society, EMBC, 2011 Annual International Conference of the IEEE. IEEE, 2011, pp. 5264-5266.

[65] "Somatic data blood glucose collection transmission device for internet of things," Mar. 27 2013, cN Patent 202,838,653. [Online]. Available: http://www.google.com/patents/CN202838653U?cl=en

[66] "Multi-parameter medical acquisition detector based on internet of things," Jun. 5 2013, cN Patent 202,960,774. [Online]. Available: https://www.google.com/patents/CN202960774U?cl=en

[67] "Internet-of-things human body data blood pressure collecting and transmitting device," Mar. 27 2013, cN Patent 202,821,362. [Online]. Available: http://www.google.com/patents/CN202821362U?cl=en

[68] "Carry-on blood pressure/pulse rate/blood oxygen monitoring location intelligent terminal based on internet of things," Apr. 17 2013, cN Patent 202,875,315. [Online]. Available: https://www.google.com/patents/CN202875315U?cl=en

[69] Y.-L. Zheng, B. P. Yan, Y.-T. Zhang, and C. C. Poon, "An armband wearable device for overnight and cuff-less blood pressure measurement," Biomedical Engineering, IEEE Transactions on, vol. 61, no. 7, pp. 2179-2186, 2014.

[70] "Patient body temperature monitoring system and device based on internet of things," Feb. 12 2014, cN Patent App. CN 201,310,482,743. [Online]. Available: https://www.google.com/patents/CN103577688A?cl=en

[71] A. J. Jara, M. A. Zamora-Izquierdo, and A. F. Skarmeta, "Interconnection framework for mhealth and remote monitoring based on the internet of things," Selected Areas in Communications, IEEE Journal on, vol. 31, no. 9, pp. 47-65, 2013.

[72] E. Gayat, A. Bodin, C. Sportiello, M. Boisson, J.-F. Dreyfus, E. Mathieu, and M. Fischler, "Performance evaluation of a noninvasive hemoglobin monitoring device," Annals of emergency medicine, vol. 57, no. 4, pp. 330-333, 2011.

[73] P. Gupta and T. Dallas, "Feature selection and activity recognition system using a single triaxial accelerometer," Biomedical Engineering, IEEE Transactions on, vol. 61, no. 6, pp. 1780-1786, 2014.

[74] S. I. Lee, E. Park, A. Huang, B. Mortazavi, J. H. Garst, N. Jahanforouz, M. Espinal, T. Siero, S. Pollack, M. Afridi et al., “Objectively quantifying walking ability in degenerative spinal disorder patients using sensor equipped smart shoes," Medical engineering \& physics, vol. 38, no. 5, pp. 442-449, 2016.

[75] E. McAdams, A. Krupaviciute, C. Gehin, A. Dittmar, G. Delhomme, P. Rubel, J. Fayn, and J. McLaughlin, "Wearable electronic systems: Applications to medical diagnostics/monitoring," in Wearable Monitoring Systems. Springer, 2011, pp. 179-203.

[76] R. Paradiso, G. Loriga, and N. Taccini, "A wearable health care system based on knitted integrated sensors," Information Technology in Biomedicine, IEEE Transactions on, vol. 9, no. 3, pp. 337-344, 2005.

[77] S. Pasche, S. Angeloni, R. Ischer, M. Liley, J. Luprano, and G. Voirin, "Wearable biosensors for monitoring wound healing," in Advances in Science and Technology, vol. 57. Trans Tech Publ, 2009, pp. 80-87.

[78] A. M. Adami, M. Pavel, T. L. Hayes, and C. M. Singer, "Detection of movement in bed using unobtrusive load cell sensors," Information Technology in Biomedicine, IEEE Transactions on, vol. 14, no. 2, pp. 481-490, 2010.

[79] W. H. Wu, A. A. Bui, M. A. Batalin, L. K. Au, J. D. Binney, and W. J. Kaiser, "Medic: Medical embedded device for individualized care," Artificial Intelligence in Medicine, vol. 42, no. 2, pp. 137-152, 2008.

[80] M.-Z. Poh, T. Loddenkemper, C. Reinsberger, N. Swenson, S. Goyal, J. Madsen, and R. Picard, "Autonomic changes with seizures correlate with postictal eeg suppression," Neurology, vol. 78, no. 23, pp. 1868-1876, 2012.

[81] Empatica, available at https://www.empatica.com/product-e4.

[82] G. D. Abowd and E. D. Mynatt, "Designing for the human experience in smart environments," Smart environments: technologies, protocols, and applications, pp. 151-174, 2005.

[83] B. Bouchard, S. Giroux, and A. Bouzouane, "A keyhole plan recognition model for alzheimer's patients: first results," Applied Artificial Intelligence, vol. 21, no. 7, pp. 623-658, 2007.

[84] E. M. Tapia, S. S. Intille, and K. Larson, Activity recognition in the home using simple and ubiquitous sensors. Springer, 2004.

[85] T. Yamazaki, "The ubiquitous home," International Journal of Smart Home, vol. 1, no. 1, pp. 17-22, 2007.

[86] T. Tamura, A. Kawarada, M. Nambu, A. Tsukada, K. Sasaki, and K.-I. Yamakoshi, "E-healthcare at an experimental welfare techno house in japan," The open medical informatics journal, vol. 1, no. 1, 2007.

[87] H. Kalantarian, B. Motamed, N. Alshurafa, and M. Sarrafzadeh, "A wearable sensor system for medication adherence prediction," Artificial intelligence in medicine, vol. 69, pp. 43-52, 2016. 
[88] Z. Pang, J. Tian, and Q. Chen, "Intelligent packaging and intelligent medicine box for medication management towards the internet-of-things," in Advanced Communication Technology (ICACT), 2014 16th International Conference on. IEEE, 2014, pp. 352-360.

[89] S.-M. S. S.-W. Kim and J.-W. Jeon, "Food contamination monitoring via internet of things," in Conference on, IEEE, 2015 , pp. 609-613.

[90] D. Georgaka, M. Mparmparousi, and M. Vitos, "Early warning systems," Hospital Chronicles, vol. 7, no. 1 Sup, p. 37, 2012.

[91] A. Anzanpour, A.-M. Rahmani, P. Liljeberg, and H. Tenhunen, "Internet of things enabled in-home health monitoring system using early warning score," in Proceedings of the 5th EAI International Conference on Wireless Mobile Communication and Healthcare. ICST (Institute for Computer Sciences, Social-Informatics and Telecommunications Engineering), 2015, pp. 174-177.

[92] M. Liaqat, V. Chang, A. Gani, S. H. a. Hamid, R. L. Ali, R. M. Haseeb, and T. Maqsood, "Towards sensor-cloud integration: A survey of enabling technologies and architectures," International Journal of Information Management, 2016.

[93] H. Dubey, J. C. Goldberg, M. Abtahi, L. Mahler, and K. Mankodiya, "Echowear: smartwatch technology for voice and speech treatments of patients with parkinson's disease," in Proceedings of the conference on Wireless Health. ACM, 2015, p. 15.

[94] K. Mankodiya, V. Sharma, R. P. Martins, I. Pande, S. Jain, N. Ryan, and R. Gandhi, "Understanding user's emotional engagement to the contents on a smartphone display: Psychiatric prospective," in Ubiquitous Intelligence and Computing, 2013 IEEE 10th International Conference on and 10th International Conference on Autonomic and Trusted Computing (UIC/ATC). IEEE, 2013, pp. 631-637.

[95] V. Sharma, K. Mankodiya, F. De La Torre, A. Zhang, N. Ryan, T. G. Ton, R. Gandhi, and S. Jain, "Spark: personalized parkinson disease interventions through synergy between a smartphone and a smartwatch," in Design, User Experience, and Usability. User Experience Design for Everyday Life Applications and Services. Springer, 2014, pp. 103-114.

[96] Texas Instruments Wireless Connectivity, available at www.ti.com/wirelessconnectivity.

[97] Health Level Seven International, available at http://www.hl7.org/index.cfm.

[98] IDepartment of Economic and Social Affairs, available at http://www.un.org.

[99] A. Dohr, R. Modre-Opsrian, M. Drobics, D. Hayn, and G. Schreier, "The internet of things for ambient assisted living," in 2010 Seventh International Conference on Information Technology. Ieee, 2010, pp. 804-809.

[100] N. Suryadevara, S. Kelly, and S. Mukhopadhyay, "Ambient assisted living environment towards internet of things using multifarious sensors integrated with xbee platform," in Internet of Things. Springer, 2014, pp. 217-231.

[101] A. J. Jara, F. J. Belchi, A. F. Alcolea, J. Santa, M. A. Zamora-Izquierdo, and A. F. Gómez-Skarmeta, "A pharmaceutical intelligent information system to detect allergies and adverse drugs reactions based on internet of things," in Pervasive Computing and Communications Workshops (PERCOM Workshops), 2010 8th IEEE International Conference on. IEEE, 2010, pp. 809-812.

[102] W. H. Organization, The world health report: World report on disability. World Health Organization, 2002.

[103] D. K. Sarji, "Handtalk: Assistive technology for the deaf," Computer, vol. 41, no. 7, pp. 84-86, 2008.

[104] P. Kumar, J. Verma, and S. Prasad, "Hand data glove: a wearable real-time device for human-computer interaction," International Journal of Advanced Science and Technology, vol. 43, 2012.

[105] Y. Li, X. Chen, J. Tian, X. Zhang, K. Wang, and J. Yang, "Automatic recognition of sign language subwords based on portable accelerometer and emg sensors," in International Conference on Multimodal Interfaces and the Workshop on Machine Learning for Multimodal Interaction. ACM, 2010, p. 17.

[106] H. Dubey, J. C. Goldberg, K. Makodiya, and L. Mahler, "A multi-smartwatch system for assessing speech characteristics of people with dysarthria in group settings," in Proceedings e-Health Networking, Applications and Services (Healthcom), 2015 IEEE 17th International Conference on, Boston, USA, 2015

[107] L. Yang, W. Li, Y. Ge, X. Fu, R. Gravina, and G. Fortino, "People-centric service for mhealth of wheelchair users in smart cities," in Internet of Things Based on Smart Objects. Springer, 2014, pp. 163-179.

[108] M. Ferati, A. Kurti, B. Vogel, and B. Raufi, "Augmenting requirements gathering for people with special needs using iot: a position paper," in Proceedings of the 9th International Workshop on Cooperative and Human Aspects of Software Engineering. ACM, 2016, pp. 48-51.

[109] The Wireless Nano Retina Eyeglasses, available at http://www.nano-retina.com/.

[110] Definition of Implants and Prosthetics. U.S. Food and Drug Administration. FDA, available at http://http://www.fda.gov/MedicalDevices/.

[111] S. S. Barold, R. X. Stroobandt, and A. F. Sinnaeve, Cardiac pacemakers and resynchronization step by step: An illustrated guide. John Wiley \& Sons, 2010.

[112] J. M. Bronstein, M. Tagliati, R. L. Alterman, A. M. Lozano, J. Volkmann, A. Stefani, F. B. Horak, M. S. Okun, K. D. Foote, P. Krack et al., "Deep brain stimulation for parkinson disease: an expert consensus and review of key issues," Archives of neurology, vol. 68, no. 2, pp. 165-165, 2011.

[113] J. Y. Lee and D. Kondziolka, "Thalamic deep brain stimulation for management of essential tremor," Journal of neurosurgery, vol. 103, no. 3, pp. 400-403, 2005.

[114] A. J. Espay, P. Bonato, F. B. Nahab, W. Maetzler, J. M. Dean, J. Klucken, B. M. Eskofier, A. Merola, F. Horak, A. E. Lang et al., "Technology in parkinson's disease: Challenges and opportunities," Movement Disorders, 2016.

[115] M. Arlotti, M. Rosa, S. Marceglia, S. Barbieri, and A. Priori, "The adaptive deep brain stimulation challenge," Parkinsonism \& related disorders, 2016.

[116] W. Maetzler, J. Klucken, and M. Horne, "A clinical view on the development of technology-based tools in managing parkinson's disease," Movement Disorders, 2016.

[117] R. Tyler, P. R. Kileny, A. K. Deshpande, S. B. Deshpande, C. Dunn, M. Hansen, and B. Gantz, "The future of cochlear implants," Scientific Foundations of Audiology: Perspectives from Physics, Biology, Modeling, and Medicine, p. 175, 2016.

[118] M. Sarrafzadeh, F. Dabiri, and H. Noshadi, "Fast behavior and abnormality detection," May 26 2016, uS Patent 20,160,148,103.

[119] Numenta, available at https://numenta.com.

[120] IBM Watson Health, available at http://www.ibm.com/smarterplanet/us/en/ibmwatson/health/.

[121] E. Smets, P. Casale, U. Grossekathoefer, B. Lamichhane, W. De Raedt, K. Bogaerts, I. Van Diest, and C. Van Hoof, "Comparison of machine learning techniques for psychophysiological stress detection," in Mindcare 2015-5th EAI International Symposium on Pervasive Computing Paradigms for Mental Health, 2015.

[122] A. Sano and R. W. Picard, "Stress recognition using wearable sensors and mobile phones," in Affective Computing and Intelligent Interaction (ACII), 2013 Humaine Association Conference on. IEEE, 2013, pp. 671-676.

[123] W. Raghupathi and V. Raghupathi, "Big data analytics in healthcare: promise and potential," Health Information Science and Systems, vol. 2, no. 1, p. 1, 2014.

[124] A. Ghazal, T. Rabl, M. Hu, F. Raab, M. Poess, A. Crolotte, and H.-A. Jacobsen, "Bigbench: towards an industry standard benchmark for big data analytics," in Proceedings of the 2013 ACM SIGMOD international conference on Management of data. ACM, 2013, pp. 1197-1208.

[125] I. A. T. Hashem, I. Yaqoob, N. B. Anuar, S. Mokhtar, A. Gani, and S. U. Khan, "The rise of big data on cloud computing: Review and open research issues," Information Systems, vol. 47, pp. 98-115, 2015.

[126] A. Bourouis, A. Zerdazi, M. Feham, and A. Bouchachia, "M-health: skin disease analysis system using smartphone's camera," Procedia Computer Science, vol. 19, pp. 1116-1120, 2013.

[127] I. A. T. Hashem, V. Chang, N. B. Anuar, K. Adewole, I. Yaqoob, A. Gani, E. Ahmed, and H. Chiroma, "The role of big data in smart city," International Journal of Information Management, vol. 36, no. 5, pp. 748-758, 2016. 
[128] T. G. Mahn, "Wireless medical technologies: Navigating government regulation in the new medical age," Fishs Regulatory \& Government Affairs Group, 2013

[129] M. Gordon, R. Henderson, J. H. Holmes, M. K. Wolters, I. M. Bennett et al., "Participatory design of ehealth solutions for women from vulnerable populations with perinatal depression," Journal of the American Medical Informatics Association, vol. 23, no. 1, pp. 105-109, 2016.

[130] G. Suciu, V. Suciu, A. Martian, R. Craciunescu, A. Vulpe, I. Marcu, S. Halunga, and O. Fratu, "Big data, internet of things and cloud convergence-an architecture for secure e-health applications," Journal of medical systems, vol. 39, no. 11, p. 141, 2015.

[131] W. Jansen, T. Grance et al., "Guidelines on security and privacy in public cloud computing," NIST special publication, vol. 800, no. 144, pp. 10-11, 2011.

[132] R. Roman, J. Zhou, and J. Lopez, "On the features and challenges of security and privacy in distributed internet of things," Computer Networks, vol. 57, no. 10 , pp. 2266-2279, 2013.

[133] McAfee, available at http://mcafee.com/.

[134] A. Ukil, J. Sen, and S. Koilakonda, "Embedded security for internet of things," in Emerging Trends and Applications in Computer Science (NCETACS), 2011 2nd National Conference on. IEEE, 2011, pp. 1-6.

[135] M. M. Hossain, M. Fotouhi, and R. Hasan, "Towards an analysis of security issues, challenges, and open problems in the internet of things," in Services (SERVICES), 2015 IEEE World Congress on. IEEE, 2015, pp. 21-28.

[136] H. Löhr, A.-R. Sadeghi, and M. Winandy, "Securing the e-health cloud," in Proceedings of the 1st ACM International Health Informatics Symposium. ACM, 2010, pp. 220-229.

[137] H. Suo, J. Wan, C. Zou, and J. Liu, "Security in the internet of things: a review," in Computer Science and Electronics Engineering (ICCSEE), 2012 International Conference on, vol. 3. IEEE, 2012, pp. 648-651.

[138] P. Pharow and B. Blobel, "Mobile health requires mobile security: Challenges, solutions, and standardization," Studies in health technology and informatics, vol. 136, p. 697, 2008.

[139] symantec, available at https://www.symantec.com/solutions/internet-of-things.

[140] WindRiver, available at http://www.windriver.com/iot/.

[141] Q. Jing, A. V. Vasilakos, J. Wan, J. Lu, and D. Qiu, "Security of the internet of things: Perspectives and challenges," Wireless Networks, vol. 20, no. 8, pp. 2481-2501, 2014

[142] Pulse Sensor Datasheet, available at http://pulsesensor.com/pages/open-hardware.

[143] W. Ng and H.-L. Lau, "Effective approaches for watermarking xml data," in International Conference on Database Systems for Advanced Applications. Springer, 2005, pp. 68-80.

[144] Redis, available at http://redis.io/

[145] Kafka, available at http://kafka.apache.org/.

[146] Mongodb, available at https://www.mongodb.com/.

[147] Apache Hadoop, available at http://hadoop.apache.org/.

[148] Dato, available at https://dato.com/.

[149] Plant, Noriega, Sonti, and Mankodiya, "Smart e-textile gloves for quantified measurements in movement disorders," in IEEE MIT Undergraduate Research Technology Conference, 2016.

[150] C. G. Goetz, W. Poewe, O. Rascol, C. Sampaio, G. T. Stebbins, C. Counsell, N. Giladi, R. G. Holloway, C. G. Moore, G. K. Wenning et al., "Movement disorder society task force report on the hoehn and yahr staging scale: status and recommendations the movement disorder society task force on rating scales for parkinson's disease," Movement disorders, vol. 19, no. 9, pp. 1020-1028, 2004.

[151] H. Dubey, A. Monteiro, L. Mahler, U. Akbar, Y. Sun, Q. Yang, and K. Mankodiya, "Fogcare: fog-assisted internet of things for smart telemedicine," Future Gen Comput Syst, 2016.

[152] A. Monteiro, H. Dubey, L. Mahler, Q. Yang, and K. Mankodiya, "Fit a fog computing device for speech teletreatments," arXiv preprint arXiv:1605.06236, 2016. 